\title{
Activités
}

17-1 | 2020

IA, robotique, automatisation : quelles évolutions pour l'activité humaine?

\section{Apprendre dans des interactions avec de multiples participants : la formation pratique des éducatrices et éducateurs de l'enfance}

Learning through complex participatory configurations: the vocational training of early childhood educators during internships

\section{Marianne Zogmal}

\section{OpenEdition}

\section{Journals}

Édition électronique

URL : http://journals.openedition.org/activites/5029

DOI : 10.4000/activites.5029

ISSN : $1765-2723$

Éditeur

ARPACT - Association Recherches et Pratiques sur les ACTivités

\section{Référence électronique}

Marianne Zogmal, «Apprendre dans des interactions avec de multiples participants : la formation pratique des éducatrices et éducateurs de l'enfance », Activités [En ligne], 17-1 | 2020, mis en ligne le 15 avril 2020, consulté le 10 décembre 2020. URL : http://journals.openedition.org/activites/5029 ; DOI : https://doi.org/10.4000/activites.5029

Ce document a été généré automatiquement le 10 décembre 2020.

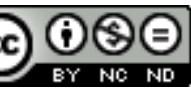

Activités est mis à disposition selon les termes de la licence Creative Commons Attribution - Pas d'Utilisation Commerciale - Pas de Modification 4.0 International. 


\title{
Apprendre dans des interactions avec de multiples participants : la formation pratique des éducatrices et éducateurs de l'enfance
}

\author{
Learning through complex participatory configurations: the vocational training \\ of early childhood educators during internships
}

Marianne Zogmal

\section{NOTE DE L'ÉDITEUR}

Article soumis le 13 mai 2019, accepté le 16 décembre 2019

1 Les métiers de la prise en charge d'autrui, de l'éducation, des soins ou du travail social, impliquent d'interagir avec les bénéficiaires du travail. Ces interactions peuvent se dérouler à deux, lors d'un entretien ou par un accompagnement individualisé. Cependant, dans beaucoup de champs professionnels, le travail auprès des bénéficiaires de la prise en charge s'effectue dans un contexte interactionnel regroupant de multiples participants. Par ailleurs, les dispositifs de formation dans les métiers de la prise en charge d'autrui prévoient généralement des stages de formation pratiques dans la situation de travail. Dans les contextes de ces formations pratiques, les apprenants se trouvent alors dans des situations où la dimension collective est particulièrement prégnante.

2 Ceci concerne notamment les professionnelles de l'éducation de l'enfance ${ }^{1}$. Pendant leur parcours de formation, les éducatrices de l'enfance formées en Suisse à un niveau d'études supérieures effectuent plusieurs stages de formation pratiques dans les structures d'accueil de la petite enfance. Elles découvrent un contexte collectif, regroupant de nombreux enfants et plusieurs professionnelles. La présence simultanée 
de multiples participants à une activité en cours constitue pour les éducatrices en formation un défi et offre des opportunités d'apprentissage. La situation de stage nécessite de savoir varier les adressages, de tenir compte des conduites de plusieurs participants pour s'insérer dans le déroulement des interactions. Il s'agit d'effectuer un travail éducatif orienté vers les enfants, tout en s'engageant dans un processus formatif. Dans la réalisation des activités éducatives auprès des enfants, les interactions avec les enfants ne peuvent pas être suspendues longuement pour aborder une dimension formative. Dès lors, les aspects éducatifs et formatifs s'imbriquent et constituent « une activité institutionnelle hybride » (Markaki-Lothe \& Filliettaz, 2017, p. 81).

3 La présence simultanée de nombreux participants, enfants et professionnelles, pose des problèmes pratiques aux étudiantes en formation. En évoquant à l'occasion d'une session de formation à l'école professionnelle la situation du stage abordée ci-après, l'étudiante en formation considère qu'il ne pas toujours simple de "trouver sa place ", et que les membres de l'équipe éducative procèdent à de fréquentes interruptions du travail : «ils sont beaucoup. en fait . cette équipe. dans l'intervention avec les autres. ils ne laissent pas forcément- . eh . les adultes continuer dans leur intervention. " A l'occasion d'un entretien collectif avec d'autres étudiantes stagiaires, elle considère aussi qu'à certains moments, les interventions de sa référente professionnelle ou d'autres professionnelles de l'équipe peuvent perturber son action : "ça m'a un peu . un peu dérangé. en fait . parce que j'avais envie de trouver moi». Ces remarques illustrent qu'en tant qu'apprenant, il n'est pas toujours aisé de s'insérer dans un contexte collectif, de participer à une activité, de maintenir sa participation, d'ajuster et de réorienter ses conduites dans des situations avec de multiples participants.

Dans des environnements interactionnels complexes regroupant de multiples participants, se former nécessite de pouvoir accéder et contribuer de manière pertinente et légitime au déroulement des interactions. Pour étudier ces situations, cet article est centré a) sur la problématique des interactions avec de multiples participants dans le champ de l'éducation de l'enfance et b) sur l'étude de l'activité des stagiaires. Il explore la manière dont les stagiaires peuvent trouver une place dans des interactions regroupant une diversité de personnes et comment elles apprennent à interagir dans un contexte professionnel complexe. Cet article s'inscrit dans une relation de complémentarité avec des recherches antérieures portant sur les situations de formation dans le contexte du travail, mais qui avaient davantage porté sur l'activité des tuteurs (Filliettaz et al. 2014).

5 Comment les interactions avec de multiples participants se déroulent-elles lors des activités éducatives menées auprès des enfants? Comment les apprenants arrivent-ils à y participer et à s'ajuster à ce qui se passe? Pour répondre à ces différents questionnements, cet article mobilise la notion de participation, qui s'inscrit au croisement de différentes traditions et perspectives théoriques. Les travaux portant sur la formation en situation de travail considèrent la participation des novices aux activités en cours comme un élément fondamental pour favoriser les apprentissages. Comment cette participation se déploie-t-elle dans les pratiques quotidiennes? Le présent article vise à étudier comment une perspective interactionnelle peut contribuer à enrichir la compréhension de la notion de participation à travers une analyse fine des dynamiques interactionnelles. En considérant que «la compétence 
interactionnelle se manifeste dans les modes de participation à l'action en cours » (Mondada, 2006, p. 95), il s'agit d'éclairer les processus déployés pour y parvenir.

6 En premier, cet article aborde les travaux s'intéressant à la formation dans des situations de travail et qui étudie les processus d'apprentissage en tant que participation à des communautés de pratique (1). Pour suivre, il présente la notion de participation dans des contextes professionnels avec de multiples participants dans une perspective interactionnelle (2). Les orientations théoriques et méthodologiques d'une perspective interactionnelle permettent notamment de présenter certaines modalités de participation (2.2.) et la notion de configuration de participation (2.3). L'analyse détaillée des données empiriques cherche ensuite à montrer la dimension dynamique de ces modalités de participation et à faire ressortir le caractère complexe des situations de formation dans des contextes de travail qui s'inscrivent dans des environnements interactionnels impliquant de multiples participants (3). En reprenant l'analyse empirique sur un plan plus général, le regard analytique porte ensuite sur les phénomènes de fluctuations dans la participation de la stagiaire. Il s'agit d'étudier comment celle-ci maintient la progressivité de l'activité, tout en s'ajustant continuellement à ce qui se passe (4). Ces différents éléments sont finalement mis en perspective pour interroger les liens entre la notion de participation et les enjeux de la formation en situation de travail (5).

\section{L'apprentissage comme participation à des communautés de pratique}

7 Selon de nombreuses recherches menées à propos de l'apprentissage en situation de travail, la formation ne saurait se réduire à une activité de transmission de connaissances. Ainsi, «apprendre ne consiste pas à assimiler des informations et des raisonnements transmis par des acteurs expérimentés, mais revient à participer de manière adéquate aux activités propres à la situation, en exploitant les ressources disponibles dans l'environnement» (Filliettaz, de Saint-Georges, \& Duc, 2008, p. 45). La notion de participation est centrale pour étudier comment les apprenants peuvent accomplir des activités de travail, s'ajuster et se coordonner avec les professionnelles présentes, s'inscrire dans un fonctionnement d'une organisation de travail. Les collectifs de travail forment des « communautés de pratique » qui tendent à partager un système d'attentes normatives et comportementales traduisant des normes, des croyances, des valeurs et des attitudes spécifiques (Lave \& Wenger, 1991 ; Wenger, 1999). Participer aux activités d'une communauté de pratiques peut ainsi permettre de se confronter aux manières d'interagir, d'accomplir des actions conjointes et de tenir compte des attentes existantes. Les apprentissages «se rapportent nécessairement à des pratiques sociales identifiables et s'inscrivent par conséquent au cœur des groupes d'individus qui s'y trouvent engagés » (Filliettaz et al., 2008, p. 53). Cependant, il ne suffit pas d'être simplement présent dans un contexte professionnel pour qu'un apprentissage se réalise :

"L'expression: "apprendre sur le tas" est de ce point de vue relativement trompeuse. On apprend certes sur le terrain, en se frottant aux situations, mais toute acquisition de routines performantes, reconnues comme telles par le milieu de travail, suppose des tâtonnements, des rectifications d'erreurs, un minimum de retour sur la manière de s'y prendre, des conseils donnés formellement ou 
informellement par quelqu'un qui maîtrise déjà les bonnes routines, etc. Bref, un

apprentissage » (Zarifian, 2001, pp. 154-155).

Élaborer la définition d'une situation, attribuer une signification à ce qui se passe et déceler les attentes normatives d'un collectif de travail ne peut s'effectuer de façon solitaire par un apprenant isolé. Il s'agit ici de processus qui se déroulent dans les interactions, se co-construisent dans une interdépendance de tous les participants dans un contexte donné. Pour apprendre à participer à des situations de travail, « le rôle des autres est déterminant" (Mayen, 2002, p. 87). L'apport d'autrui introduit une médiation entre l'apprenant et le contexte professionnel qu'il découvre. Les interventions d'autrui sont parfois bienvenues pour les stagiaires, ou, à d'autres moments, dérangeantes. Dans tous les cas, elles constituent des «ruptures» dans l'activité de la personne en formation, entre ce qu'elle prévoit et ce qui se réalise effectivement (Pastré, Mayen, \& Vergnaud, 2006). Les phénomènes de médiation par des praticiens expérimentés peuvent se dérouler dans des dispositifs de formation institués ou de façon plus informelle dans des collectifs de travail. Dans la réalité des stages professionnels, le tutorat n'est pas exercé uniquement par une personne désignée, revêtant un statut de tuteur, mais s'accomplit également de façon distribuée, par l'ensemble des acteurs présents. Ces phénomènes d'un tutorat distribué influencent sur les modalités de participation des étudiantes en formation.

\section{Une perspective interactionnelle sur des contextes professionnels avec de multiples participants}

9 Dans une perspective interactionnelle, la notion de participation renvoie aux modalités d'accès et de contributions des individus aux interactions dans lesquelles ils prennent part. Les interactions sont centrales dans les métiers de la prise en charge d'autrui, ainsi que dans les pratiques de formation professionnelle qui y préparent. Interagir contribue à s'ajuster mutuellement, à se coordonner, à réaliser des activités conjointes, mais également à faciliter la participation de chacun, à rendre visibles les éléments saillants d'un contexte donné.

Pour étudier ces phénomènes, la perspective interactionnelle adoptée s'appuie sur une description ethnographique des pratiques réelles ainsi que sur un recueil d'enregistrements audio-vidéo de situations de travail et de formation. Dans cet article, l'analyse des interactions vise à mieux comprendre les enjeux de travail et de formation et offre des moyens théoriques et méthodologiques pour étudier les processus en jeu. Pour documenter la dynamique de l'activité dans son déploiement séquentiel et les modalités de participation des différents interactants, les interactions sont étudiées comme des activités situées, accomplies de façon collective et au moyen de multiples ressources sémiotiques (Filliettaz, 2014 ; Filliettaz et al., 2008). L'analyse des conduites interactionnelles, dans l'ordre où elles se présentent, permet de caractériser les interactions dans leurs conditions d'accomplissement temporellement ordonnées, et dans leur déroulement étape par étape. En ce qui concerne l'aspect collectif, il est inhérent à toute situation interactionnelle ce qui est notamment souligné par les tenants de l'analyse conversationnelle (Sacks, Schegloff, \& Jefferson, 1974). Cependant, dans une large mesure, leurs analyses portent "sur une interaction [talk-ininteraction] homogène et focalisée » (Grosjean \& Traverso, 1998, p. 52). Les situations avec de multiples participants complexifient le déroulement interactionnel et son 
analyse. En se centrant sur ces contextes complexes, il semble essentiel de tenir compte des éléments liés à la définition de la situation et aux phénomènes d'adressage, de focalisation et de figuration.

\subsection{Les modalités de participation dans des situations d'interaction avec de multiples participants}

\section{a) La définition de la situation}

11 Participer à une interaction, à une activité collective, nécessite d'identifier ce qui se passe. Les travaux d'Erving Goffman portant sur le « cadrage de l'expérience » abordent comment les participants à une interaction s'organisent pour délimiter « une définition de situation » (Goffman, 1991, p.19). Les définitions de la situation ne se font que rarement de façon explicite, mais se construisent dans le cours de l'interaction et se présentent donc «comme un produit de l'interaction» (Vion, 2000, p. 104). Les modalités de participation des interactants constituent des « définitions incarnées dans le formatage spécifique des actions des participants» (Mondada, 2017, p. 323). Les cadres de l'expérience revêtent un aspect de "vulnérabilité » (Goffman, 1991) lors de leur mobilisation dans les interactions situées. La pluralité des participants renforce l'aspect incertain et "vulnérable » des cadrages de l'expérience et la complexité des modalités pour définir ce qui est en train de se passer. Il est possible que plusieurs cadres émergent simultanément et leur pertinence varie selon le contexte. Les différents participants à une interaction comprennent parfois différemment les définitions d'une situation ou se méprennent sur ce qui se passe. Dans certaines situations, plusieurs cadres s'imbriquent ou se superposent et les différents participants à une interaction peuvent s'y inscrire de façon diverse. Ainsi, les cadres de l'expérience sont amenés à donner lieu à des négociations, à se modifier, à être validés ou contestés et fluctuent dans le déroulement des interactions.

\section{b) L'adressage}

12 L'aspect fluctuant des interactions avec de multiples participants est également abordé par les travaux de Goffman (1987) qui s'intéressent au «cadre participatif » d'une interaction et au statut participatif de chacun des participants, regroupés dans un espace perceptif commun. Pour interagir, l'un s'adresse à l'autre. Un participant à l'interaction peut s'adresser à l'ensemble des interactants présents, s'adresser individuellement à l'un, écouter un autre. De même, les autres interactants varient leurs adressages. La " position énonciative » de chacun des interactants se modifie donc sans cesse et peut notamment différencier des participants ratifiés ou non (Goffman, 1987, p. 141). Un interactant peut rendre clairement manifeste à qui il s'adresse et constitue ainsi son interlocuteur en tant que participant ratifié, adressé directement. A certains moments, des énoncés peuvent être adressés à l'un des interactants, mais concerner un autre des participants présents. Il s'agit alors d'un participant ratifié et adressé indirectement. Certains participants sont des témoins non ratifiés, lorsqu'ils surprennent ou épient une interaction. Ces distinctions entre adressages directs et indirects constituent des catégories distinctes. Les travaux de Traverso considèrent ces différentes positions énonciatives «en termes de gradualité » et introduisent notamment un " degré de ratification » et un " degré d'adresse » (Traverso, 1997). Les travaux de Goodwin (2007) élargissent également les travaux de Goffman et dépassent 
une typologie des participants entre locuteurs et destinataires. En s'intéressent aux phénomènes de co-construction entre co-interactants, les recherches de Goodwin abordent les formes d'actions déployées de façon dynamique par les participants à l'interaction, pour se rendre mutuellement manifeste leur compréhension des conduites interactionnelles de chacun. Dans les interactions situées, les participants élaborent, co-construisent et négocient les positions d'énonciation, de destinataire ou de témoin et identifient de façon située qui s'adressent à qui. Ainsi donc, les fluctuations des adressages et la complexité des positions d'énonciation augmentent dans des situations avec de multiples participants.

\section{c) La focalisation}

De façon complémentaire à ces positions énonciatives, les situations d'interactions avec de multiples participants peuvent donner lieu à une ou à plusieurs activités qui s'y déroulent, ainsi que se scinder ou non dans plusieurs foyers interactionnels.

Parfois, tous les interactants accomplissent une seule activité, menée de façon collective. A d'autres moments, plusieurs activités se déroulent dans un même contexte interactionnel. Une telle multiactivité peut s'effectuer de manière simultanée, dans un régime " parallèle » (Mondada, 2017, p. 340). Une partie des interactants s'oriente vers une activité, tandis que les autres interactants se centrent sur une autre activité. Une multiactivité peut également s'accomplir par des micro-alternances successives, dans un régime "imbriqué » (op.cit., p.340). Si les différentes activités menées simultanément entrent en compétition et demandent une focalisation exclusive, cela porte à la reprise d'une « mono-activité » (op.cit., p. 340).

Par ailleurs, des situations de multiactivités qui se déroulent simultanément et de façon parallèle peuvent instaurer plusieurs foyers d'interaction par une scission du cadre participatif. Les recherches en analyse conversationnelle qualifient de tels phénomènes de scissions en tant que "schismes » (Egbert, 1997a, 1997b ; Sacks et al., 1974; Simmel, 1902 ; Traverso, 2012). Les travaux d'Egbert portent sur des schismes conversationnels dans le contexte de repas en famille ou entre amis. Lors de situations réunissant au moins quatre participants, une interaction se scinde en deux foyers interactionnels. Dans chacun de ces foyers, une interaction se déroule en parallèle et comporte un ordre séquentiel non concurrentiel. Selon les travaux d'Egbert (1997a, 1997b), la possibilité d'introduire des schismes est "induite» lorsqu'un des participants propose une nouvelle thématique, par un "schisming induction turn ». Un schisme s'instaure si un participant s'aligne sur cette offre et dans le cas où deux autres participants poursuivent l'interaction en cours. Dans les situations de travail, il semble essentiel de tenir compte de la réalisation de l'activité professionnelle et d'inclure les aspects multimodaux dans les analyses menées. L'importance des phénomènes multimodaux est soulignée par les auteurs abordant la notion de « schisme interactionnel » (MarkakiLothe \& Filliettaz, 2017).

Dans des situations où plusieurs activités et une scission du cadre participatif émergent, chaque interactant peut se focaliser de façon spécifique. La focalisation des participants à l'interaction s'accomplit à travers l'engagement dans une ou plusieurs activités, ou dans un ou plusieurs des foyers interactionnels présents. En ce qui concerne les activités en cours, les participants à l'interaction peuvent se focaliser sur l'une ou l'autre des activités en cours, par des «engagements symétriques ou asymétriques, convergents ou divergents» (Mondada, 2017, p. 351). Concernant les 
foyers interactionnels émergeant, les interactants peuvent ne s'engager dans aucun de ces foyers, dans un seul ou dans plusieurs. Dans le premier cas, les interactants se trouvent dans des situations de « co-présence non focalisées (...) où chacun vaque à ses occupations sous le regard des autres » (Grosjean, 1995, p. 33). À travers l'engagement dans un seul foyer interactionnel, les interactants s'orientent vers une monofocalisation. Finalement, l'engagement dans plusieurs foyers interactionnels peut donner lieu à des formes de polyfocalisations (Grosjean \& Traverso, 1998).

\section{d) La figuration}

17 Les modalités de participation s'élaborent par les phénomènes d'adressage et de focalisation dans le cadre d'une situation d'interaction et à travers la réalisation d'activités conjointes. Les fluctuations des formes d'adressage et de focalisation s'accomplissent en fonction des attentes normatives portant sur ce qui est considéré comme cohérent et pertinent dans une situation donnée. De façon contextualisée, les participants à l'interaction négocient la légitimité de leurs engagements réciproques. Dans des interactions avec de multiples participants, ces processus de négociation sont complexes et nécessitent de ne pas "perdre la face" (Goffman, 1974, p. 15). Les interactants accomplissent un travail de « figuration " pour maintenir une image de soi positive (face). Ces phénomènes ont été abordés par les travaux de Goffman (1974) qui considèrent que "la figuration sert à parer aux "incidents", c'est-à-dire aux événements dont les implications symboliques sont effectivement un danger pour la face » (p. 15). Les recherches menées par Brown et Levinson (1978) poursuivent les travaux portant sur le travail de figuration et considèrent que la notion de face peut être abordée en distinguant une «face positive » et une «face négative ». En résumant cette perspective, il est possible de considérer que « la "face positive" renvoie au désir de chacun d'entre nous d'être reconnu et apprécié par autrui, tandis que la "face négative" désigne notre désir de préserver un territoire et d'éviter de se voir menacé ou empêché dans nos actions (Durand, 2017, p. 81). Dans des situations d'interaction avec de multiples participants, la préservation de son champ d'action est un élément primordial et implique donc un travail de figuration de la part des différents participants à l'interaction.

\subsection{La notion de configuration de participation}

18 Dans les analyses menées dans cette contribution, la notion de configuration de participation permet d'englober «les ensembles de droits et d'obligations que contractent les participants à l'interaction, selon les modalités par lesquelles ils s'engagent dans les activités conjointes qui les rassemblent» (Durand, Trébert, \& Filliettaz, 2015). De façon située et intrinsèquement transitoire, une configuration de participation délimite des modalités de participation, liées à la définition de la situation, à l'adressage, à la focalisation et au travail de figuration.

19 Dès lors, l'analyse d'une configuration de participation située peut s'effectuer à travers l'analyse des ingrédients qui la composent. Dans des situations d'interaction avec de multiples participants, la complexité de différents phénomènes est importante et parfois les analyses montrent qu'une "appréhension globale des situations est irréalisable " (Grosjean \& Lacoste, 1999, p. 64). Un travail d'analyse de telles configurations de participation pose donc des difficultés méthodologiques. Cependant, 
les participants à une interaction parviennent à négocier et à co-construire leurs modalités de participation. Comment les participants à une interaction s'organisent-ils pour s'insérer dans des contextes fluctuants? En se basant sur la notion d'«accountability " (Garfinkel, 1967), il s'agit de considérer que les interactants se rendent mutuellement manifestes les éléments pertinents dans une situation d'interaction donnée. Selon une telle perspective, les participants se voient confrontés à des "problèmes pratiques" à résoudre. Pour ce faire, ils "produisent des formes organisées d'actions, des ethnométhodes, qu'ils rendent visibles à travers les comportements qu'ils adoptent et ceux qu'ils reconnaissent comme légitimes auprès de leurs participants » (Filliettaz, 2018, p. 19).

L'analyse des phénomènes interactionnels ne cherche pas à déceler le vécu subjectif d'un des participants, ses intentions ou ses motivations, ni à se positionner en extériorité pour proposer un regard analytique visant l'objectivité. Une perspective d'analyse interactionnelle " endosse le point de vue des participants au moment où ils s'adressent mutuellement l'un à l'autre et où ils s'appliquent, "à la deuxième personne" pourrait-on dire, à rendre leurs comportements mutuellement interprétables " (Filliettaz, 2018, p. 55). Cette orientation du regard analytique sur les conduites rendues mutuellement manifestes et interprétables permet de s'intéresser aux problèmes pratiques rencontrés par les participants à une interaction dans les situations réelles. Dans un contexte de travail et lors d'un stage de formation professionnelle, les apprenants ne peuvent généralement pas interrompre leur activité afin de susciter un entretien en vue de compléter leur compréhension de la situation. Ils sont bien confrontés à la nécessité de construire leur point de vue à partir des indices de compréhension observables. De même, les formateurs ne peuvent pas facilement interroger les stagiaires sur leur vécu, leurs intentions ou leurs objectifs, in situ. En adoptant une perspective interactionnelle, la démarche de recherche est nécessairement amenée à devoir affiner l'analyse des conduites observables et à interroger comment les participants s'orientent et s'ajustent mutuellement, avec les ressources disponibles dans l'accomplissement de leur activité.

21 Les procédés mobilisés par les interactants pour interagir s'inscrivent dans la séquentialité et la simultanéité du déroulement interactionnel. Les travaux en analyse conversationnelle se sont intéressés à l'ordre séquentiel de l'interaction. L'analyse de l'enchaînement des énoncés et des conduites interactionnelles permet de faire ressortir comment les participants rendent mutuellement visibles certaines significations et vers quels indices ils s'orientent. L'aspect temporellement ordonné du langage verbal y contribue. De façon complémentaire à la prise en compte de l'ordre séquentiel de l'interaction, les approches multimodales se sont intéressées aux effets de simultanéité. Les différents modes sémiotiques, comme les gestes, les regards, les positionnements ou l'utilisation d'objets, s'imbriquent dans le langage verbal, contribuent à faire émerger une signification et complètent, enrichissent ou modulent le sens des mots. Ces différents aspects de la multimodalité amènent un élément de simultanéité, même dans des interactions focalisées se déroulant dans un seul foyer interactionnel. Les phénomènes de simultanéité sont également ordonnés pour s'inscrire dans le processus de "meaning-making» (Kress, Jewitt, Ogborn, \& Charalampos, 2001) à travers « une série de simultanéités organisées séquentiellement » (Mondada, 2017, p. 334). Dès lors, il est possible de considérer que les interactions s'accomplissent par des phénomènes de séquentialité et de simultanéité. Les ressources multimodales permettent d'enrichir la signification d'une activité monofocalisée. Les aspects de simultanéité sont encore 
renforcés et deviennent indispensables pour pouvoir s'engager simultanément dans plusieurs activités (Mondada, 2017, p. 321) ou plusieurs foyers interactionnels.

Dans les pratiques ordinaires et notamment dans les contextes regroupant plusieurs participants, les différentes modalités de participation à une interaction s'imbriquent, se succèdent, surgissent, se résorbent, se transforment ou se maintiennent. Une analyse des interactions ne peut donc se limiter à définir des éléments typiques des configurations de participation, mais prend en compte comment les participants se rendent mutuellement manifestes les éléments qu'ils considèrent comme saillants dans un contexte donné.

\section{Animer une activité éducative dans un contexte collectif}

Dans le champ de l'éducation de l'enfance, les éducatrices en formation suivent un stage d'une durée de plusieurs mois, lors de leur troisième et dernière année de formation. Lors d'un tel stage, les stagiaires prennent en charge l'animation de différentes activités éducatives. Le présent article vise à étudier certains éléments d'une telle activité afin de décrire comment les interactions avec de multiples participants se déroulent lors des activités éducatives menées.

Les données empiriques qui alimentent cet article sont issues d'une recherche centrée sur la construction et le développement des compétences professionnelles dans le champ des métiers de la petite enfance. Des observations et des enregistrements audiovidéo d'activités de travail ont été réalisés lors des stages de pratique professionnelle de trois étudiantes en formation (Filliettaz, Rémery, \& Trébert, 2014). Ces stages se déroulaient dans des institutions de la petite enfance en Ville de Genève, accueillant des enfants d'âge variable, oscillant entre 6 mois et 4 ans. Lors de leurs stages, les éducatrices de l'enfance en formation sont accompagnées par des référentes professionnelles. Un tel accompagnement constitue une forme de tutorat qui permet d'instaurer une distance réflexive par rapport à l'activité menée en interrogeant ce qui se passe dans une situation singulière. Lors du recueil des données, un binôme formé par une étudiante stagiaire et de sa référente professionnelle a été observé au sein de chacune des institutions. La durée globale des activités éducatives observées et enregistrées sous forme de films audio-vidéo se chiffre à 21 h 30 (voir Tableau 1).

Tableau 1 : Données empiriques recueillies. Table 1: Empirical data

\begin{tabular}{|l|r|r|r|r|}
\hline Stage & $\begin{array}{l}\text { Stagiaire } \\
\text { S1 }\end{array}$ & $\begin{array}{l}\text { Stagiaire } \\
\text { S2 }\end{array}$ & $\begin{array}{l}\text { Stagiaire } \\
\text { S3 }\end{array}$ & $\begin{array}{l}\text { Totaux des } \\
\text { trois } \\
\text { stagiaires }\end{array}$ \\
\hline Stage de 1ère année & $3 \mathrm{~h} 07$ & $4 \mathrm{~h} 14$ & $4 \mathrm{~h} 25$ & $11 \mathrm{~h} 46$ \\
\hline Stage de 3ème année & $3 \mathrm{~h} 13$ & $2 \mathrm{~h} 58$ & $3 \mathrm{~h} 33$ & $9 \mathrm{~h} 44$ \\
\hline $\begin{array}{l}\text { Stage 1ère et 3ème année } \\
\text { Total activités }\end{array}$ & $6 \mathrm{~h} 20$ & $7 \mathrm{~h} 12$ & $7 \mathrm{~h} 58$ & $21 \mathrm{~h} 30$ \\
\hline
\end{tabular}

L'analyse menée se centre sur une séquence issue d'une situation de stage de troisième année de formation d'une des stagiaires (S1). Dans de nombreuses situations observées, et notamment pendant la première année de formation, les stagiaires prennent en 
charge un nombre restreint d'enfants et sont accompagnées uniquement par leur référente professionnelle. Il s'agit ici de contextes ajustés de façon spécifique à la situation de formation et qui permettent aux stagiaires d'expérimenter la réalisation d'activités éducatives dans des conditions aménagées. Progressivement, lors de la troisième année, les éducatrices en formation sont confrontées à des contextes " ordinaires" regroupant de multiples participants. Ces situations sont rencontrées principalement lors du stage de troisième année de la stagiaire $\mathrm{S} 1$.

Le choix de la séquence analysée dans cet article se porte ainsi sur une situation lors de laquelle la stagiaire ne se trouve pas uniquement en présence de sa référente professionnelle, mais également d'une éducatrice expérimentée. Il s'agit là d'un contexte de formation en situation de travail emblématique, que rencontrent très fréquemment les étudiants au cours de leurs stages. L'analyse vise à faire ressortir le caractère distribué du tutorat ainsi que la complexité d'une telle situation pour les apprenants.

Le stage professionnel se déroule dans un groupe de 16 enfants âgés de deux à trois ans. La stagiaire (ci-après: S) est accompagnée par une référente professionnelle expérimentée. Pour la matinée filmée, la stagiaire a conçu et préparé une activité de bricolage. Pour introduire la thématique du bricolage, elle a présenté aux enfants l'histoire d'un dinosaure multicolore, à l'aide d'un livre de littérature enfantine. Le bricolage consiste ensuite à coller des bandelettes de couleur sur du papier noir, découpé en forme de dinosaure. Lors de la réalisation de l'activité de bricolage, des enfants rejoignent la stagiaire, l'un après l'autre. Lors du début de la séquence choisie, six enfants (B, C, D, F, G, H) sont installés autour de la table du bricolage avec la stagiaire. Un enfant (A) a déjà fait le bricolage le jour précédent et observe ce qui se passe en étant debout à côté de la table. Le nombre d'enfants participant à l'activité dépasse ce que la stagiaire a anticipé et il manque des supports de dinosaures. La référente professionnelle $(\mathrm{R})$ a pris l'initiative de découper des formes de dinosaures. Elle est positionnée debout, à côté de la table, en train de découper.

Figure 1 : Participants à l'activité éducative.

Figure 1: Participants in the educational activity

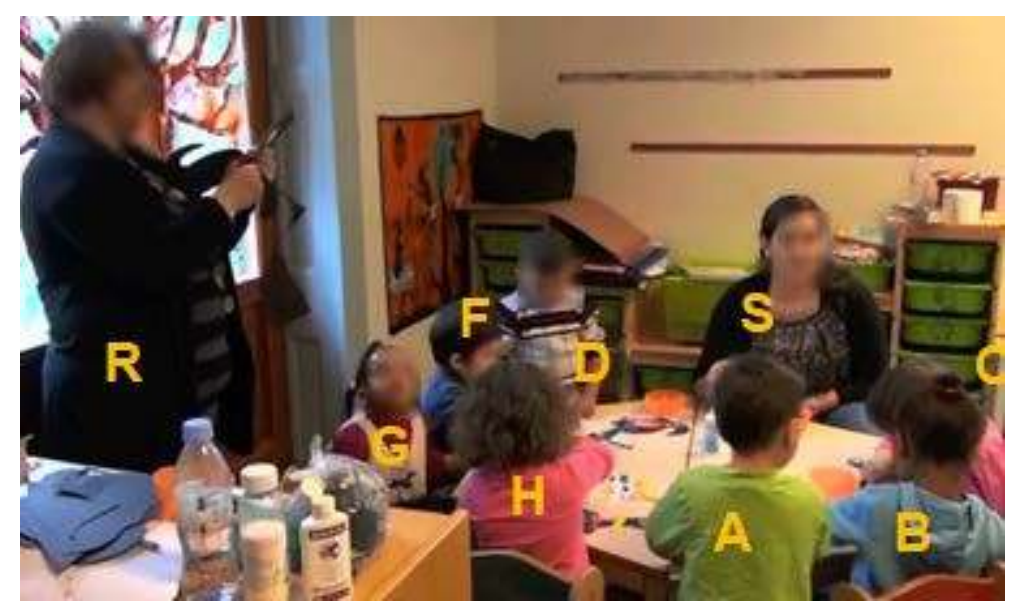

La durée de la séquence étudiée est délimitée par la durée de présence d'une des éducatrices du groupe, pendant l'animation de l'activité menée par la stagiaire. La séquence commence avec l'arrivée de l'éducatrice $(\mathrm{E})$ en question, qui revient de sa pause, et se clôture par son départ. L'arrivée de l'éducatrice va déclencher une série 
successive de changements des modalités de participation. Afin de faciliter l'analyse, la séquence est découpée en neuf extraits. Les extraits sont délimités, à chaque fois que les formes d'adressage et de focalisation des participants se modifient. La durée des différents extraits est dès lors variable, comme cela est représenté schématiquement (voir longueur des extraits dans la figure 2) :

Figure 2 : Répartition et durée temporelle des extraits 1-9 (durée totale de la séquence 1 min $52 \mathrm{~s}$ ) Figure 2: Distribution and temporal duration of extracts 1-9 (total duration of the sequence $1 \mathrm{~min}$ 52sec)

\begin{tabular}{|l|l|l|l|l|l|l|l|l|}
\hline 1 & 2 & 3 & 4 & 5 & 6 & 7 & 8 & 9 \\
\hline
\end{tabular}

Chacun de ces extraits est analysé ci-après de façon successive, avant que l'analyse vise à aborder l'ensemble de la séquence et les transformations des modalités de participation et s'intéresse aux formes d'engagement de la stagiaire dans la situation analysée. Les extraits sont transcrits en suivant des conventions de transcription développées par des approches de l'analyse interactionnelle (Filliettaz, 2018, pp. 40-51) et sont résumées en annexe.

Le premier extrait se déroule lors de l'arrivée de l'éducatrice E. tandis que la stagiaire s'oriente brièvement vers l'éducatrice $\mathrm{E}$, les enfants continuent de s'engager dans l'activité de bricolage.

Extrait 1

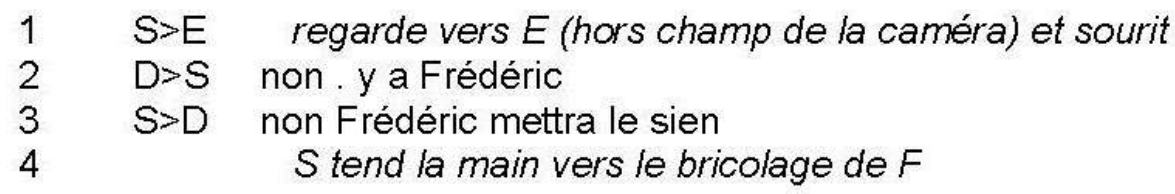

31 Un des enfants (D) interpelle la stagiaire pour lui parler du bricolage d'un autre enfant (F). La stagiaire répond à D. Dans ce premier extrait, les enfants restent focalisés sur l'activité collective du bricolage. Seule la stagiaire montre de façon fugitive qu'elle tient compte de l'arrivée de l'éducatrice, en la regardant et en lui adressant un sourire. De façon transitoire, la stagiaire varie ses adressages et s'engage dans une multiactivité, focalisée vers l'activité de bricolage ainsi que vers les autres participants présents dans l'espace commun.

Dans l'extrait suivant, la stagiaire poursuit ses échanges avec les enfants. A ce moment, l'enfant A se désengage de sa posture d'observation de l'activité en cours et s'approche de l'éducatrice qui vient d'arriver : 


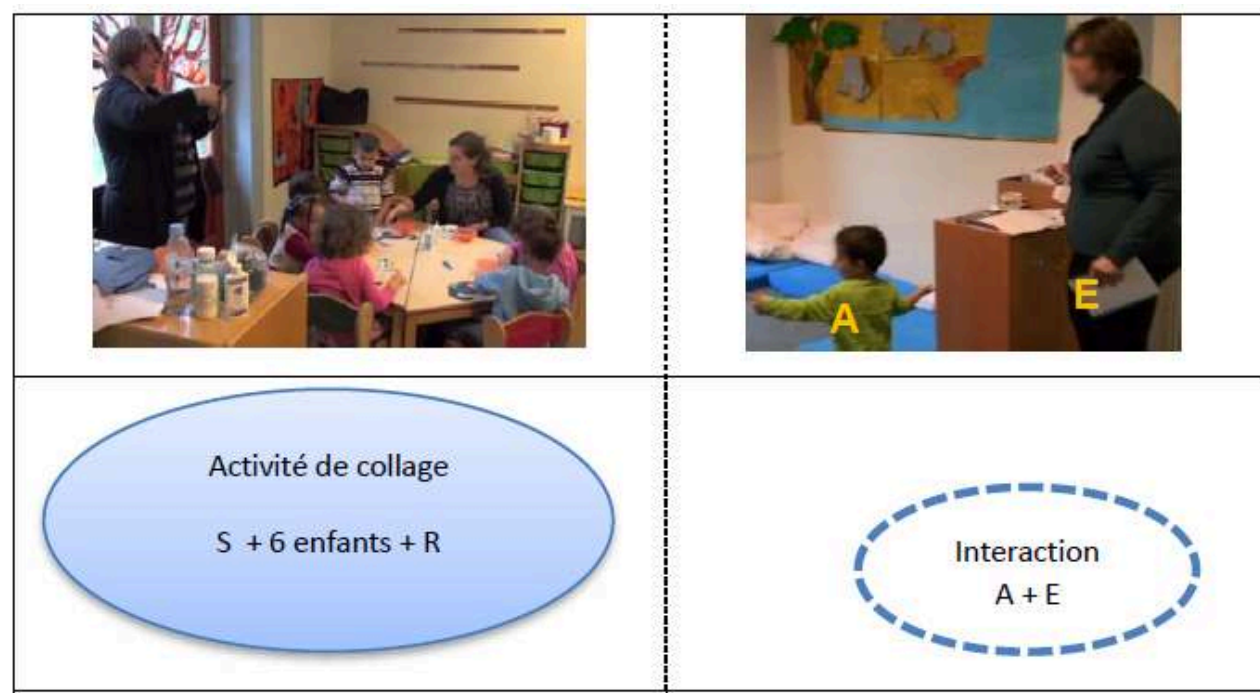

Le déplacement de A initie une scission de la participation qui peut être considérée comme un schisme (Egbert, 1997a, 1997b). Ce schisme s'instaure par l'alignement de l'éducatrice $\mathrm{E}$ sur l'interaction initiée par $\mathrm{A}$, et la poursuite de l'activité collective menée par la stagiaire et les enfants installés autour de la table. L'adressage de ses énoncés et la focalisation de la stagiaire restent orientés vers l'activité de bricolage des enfants. Deux foyers interactionnels s'instaurent en parallèle, comme cela ressort du schéma ci-dessous :

Extrait 2 : Scission de la participation. Extract 2: Participation split

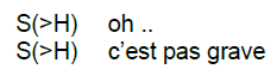

A, dans son interaction avec l'éducatrice $\mathrm{E}$, recourt brièvement au langage verbal (1. 10) et à différentes ressources multimodales, à travers son déplacement, l'orientation de ses regards et son geste de pointage. $\mathrm{E}$ vise à attribuer une signification aux conduites de A. Pour ce faire, elle montre qu'elle a observé que A n'est pas installé à table et qu'«il n'y a plus de place» (1.15). Le caractère hypothétique de cette attribution de signification se marque par la prosodie de ses énoncés, son haussement d'épaules (1.14), et le regard orienté vers A. Cet adressage direct à A contient cependant également un adressage indirect aux professionnelles présentes. De façon plus manifeste, ceci ressort lors de l'extrait suivant par l'orientation du regard de l'éducatrice en direction de la référente professionnelle :

\section{Extrait 3}

$$
\begin{array}{ll}
\mathrm{S}(>\mathrm{H}) & \text { oh .. } \\
\mathrm{S}(>\mathrm{H}) & \text { c'est pas grave }
\end{array}
$$

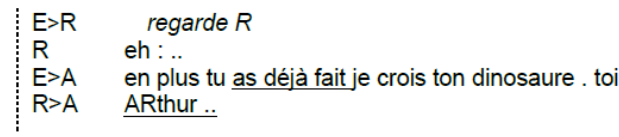


l'éducatrice $\mathrm{E}$ cherche à comprendre ce que $\mathrm{A}$ est en train de faire, elle ne s'adresse cependant pas à la stagiaire mais regarde la référente professionnelle. Celle-ci s'aligne immédiatement et s'oriente vers le foyer interactionnel regroupant $\mathrm{E}$ et $\mathrm{A}$. Cet alignement rapide fait ressortir la porosité entre les différents foyers interactionnels et l'engagement de la référente professionnelle dans une multiactivité. Elle découpe des formes de dinosaure, tout en observant l'activité de bricolage et l'interaction entre A et E. La référente annonce sa prise de parole par une vocalise (1.17). Elle se trouve hors champ de la caméra, à ce moment-là, et il n'est pas visible si elle donne de nouveaux indices portant sur des significations possibles des conduites de A. L'analyse du déroulement interactionnel montre cependant que l'éducatrice $\mathrm{E}$ se réoriente et s'adresse à $\mathrm{A}$ : «en plus tu as déjà fait, je crois ton dinosaure» (1.18). En chevauchement, la référente interpelle A par son prénom (1. 19). Pendant ces échanges entre la référente professionnelle, $\mathrm{E}$ et $\mathrm{A}$, la stagiaire répond à un des enfants $(\mathrm{H})$ qui l'avait interpellé auparavant (1.15) et continue à se focaliser sur le collage effectué (1. 16-17). La scission du cadre de participation perdure ainsi.

L'extrait suivant montre comment la référente professionnelle, puis la stagiaire s'adressent à A :

\section{Extrait 4}

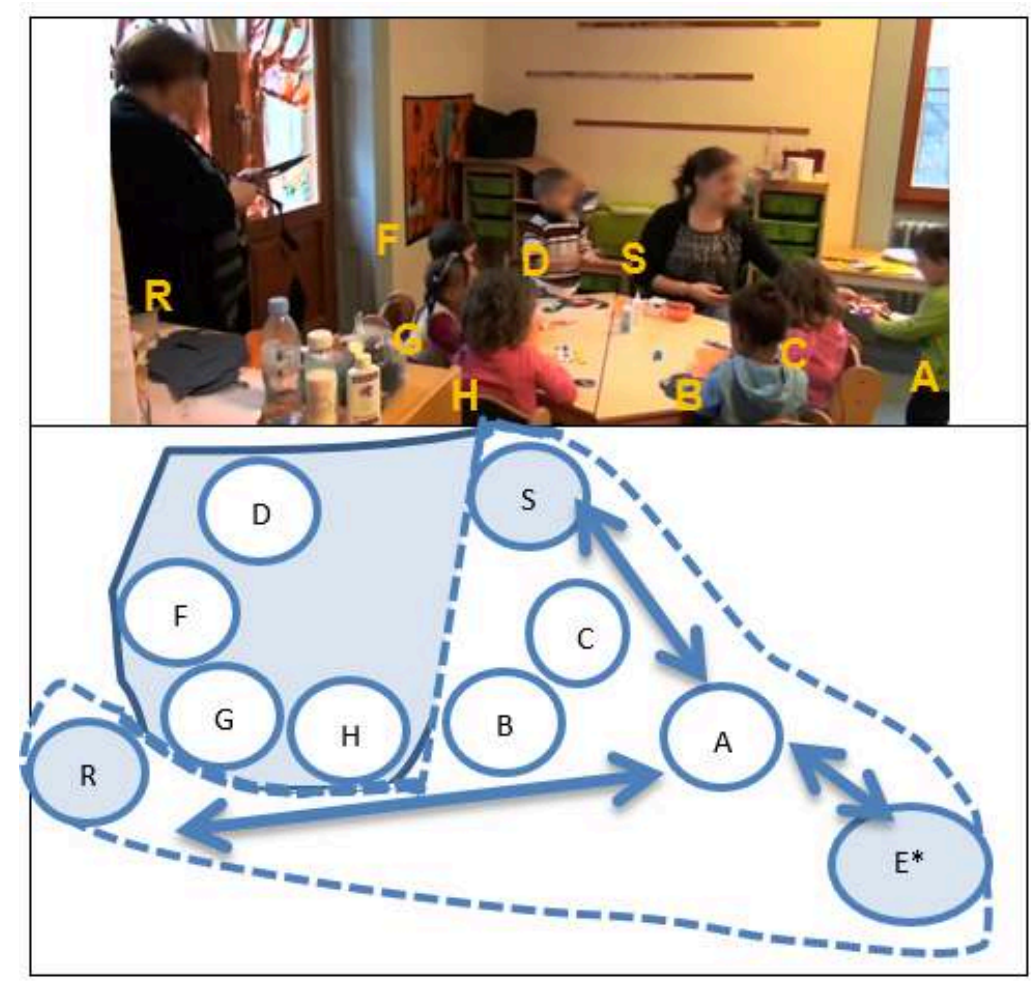

La référente interpelle $\mathrm{A}$ pour lui proposer de montrer son dinosaure à l'éducatrice $\mathrm{E}$ (1.20). A ce moment-là, la stagiaire s'adresse également de manière directe à $\mathrm{A}$ ("viens », 1.21) et commence à chercher le bricolage de A qui se trouve sur un meuble derrière elle. L'éducatrice s'aligne sur l'énoncé de la référente professionnelle et s'adresse à $\mathrm{A}$ ( « tu me le montres / il est où ton dinosaure », 1.23). A avance vers la stagiaire et prend le dinosaure que celle-ci lui tend (1.26). Les trois professionnelles 
s'adressent successivement à $\mathrm{A}$ pour l'inciter à venir montrer son dinosaure à E. Elles ne s'adressent pas directement l'une à l'autre, mais interagissent de façon indirecte par leurs énoncés adressés à $\mathrm{A}$. Les interactions focalisées vers $\mathrm{A}$ attirent l'attention de certains des enfants (B, C) qui suspendent leur activité de bricolage pour observer A.

Pendant un court moment, le foyer interactionnel focalisé sur l'accomplissement de l'activité collective du bricolage subsiste sans qu'une des professionnelles ne s'y engage. Ainsi, un des enfants s'adresse à la stagiaire à plusieurs reprises pour lui signifier qu'il a de la colle sur les mains, sans que celle-ci ne lui réponde ou s'oriente vers lui (1. 21-25). Les interactions focalisées sur A par les trois professionnelles forment ainsi une sorte de décrochage de l'activité de bricolage. De façon conjointe, l'éducatrice, la référente et la stagiaire rendent ainsi manifeste que $\mathrm{A}$ n'a certes plus de place à table et qu'il a déjà réalisé le bricolage, mais qu'elles peuvent valoriser sa participation antérieure à l'activité de collage. Cette réorganisation des modalités de participation où une grande partie des participants se focalise vers A ressort du schéma ci-dessous :

Extrait 4 : Focalisations sur les interactions orientées vers $A$.

Extract 4: Focus on interactions oriented to $A$
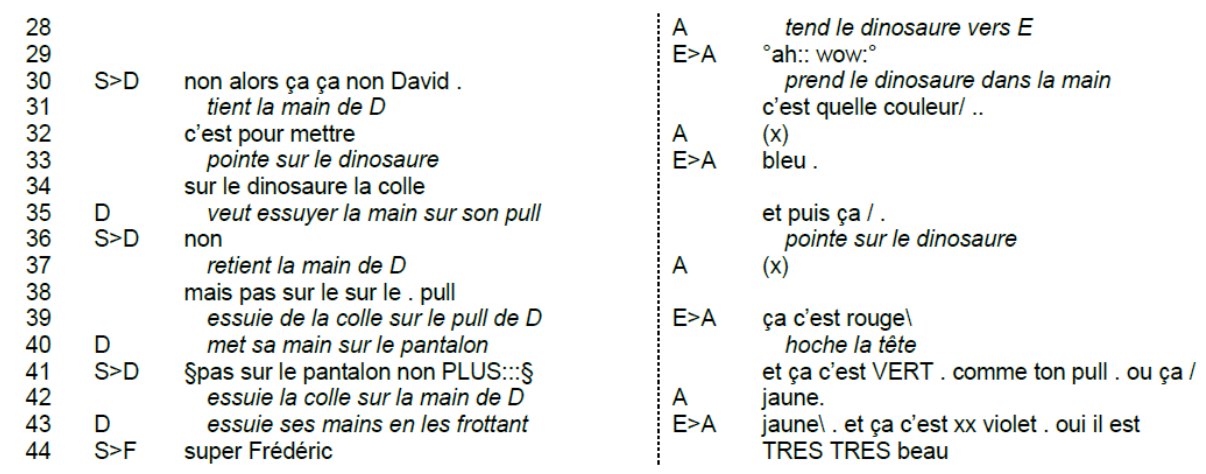

* hors champ de la caméra

Dès que la stagiaire a donné le dinosaure à $\mathrm{A}$, celui-ci revient vers l'éducatrice $\mathrm{E}$. La stagiaire se focalise à nouveau vers le bricolage et intervient auprès de $\mathrm{D}$ qui a de la colle sur les mains. Une nouvelle scission du cadre participatif se met en place : 


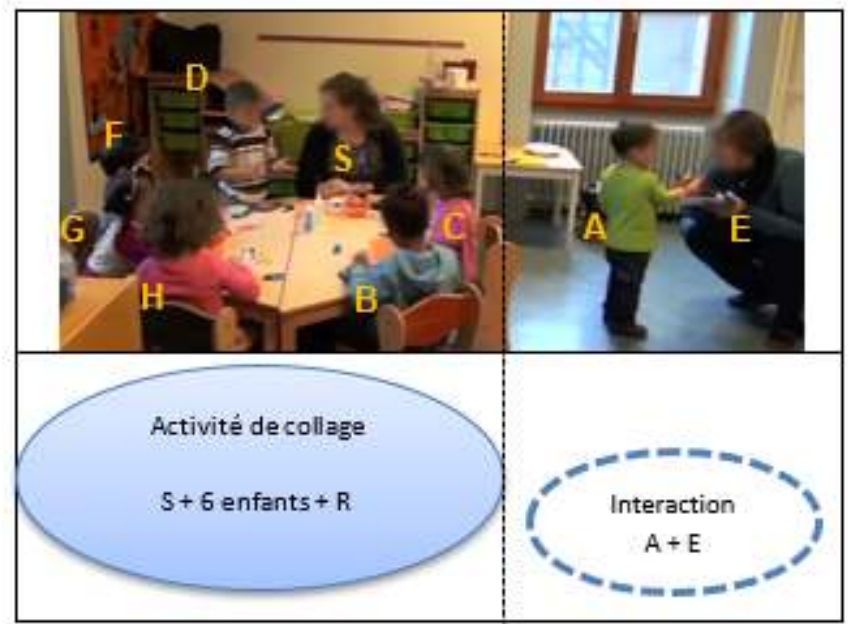

40 A montre son dinosaure à l'éducatrice E. Celle-ci le valorise en accentuant les évaluations positives («wow», 1.29). E lui parle cependant à voix basse. L'interaction entre A et elle se déroule ainsi dans un aparté, sans concurrencer la poursuite de l'activité de bricolage. E interroge A sur la désignation des couleurs des papiers collés sur le dinosaure. A lui répond d'abord de façon presque inaudible (1. 32 et 37), avant de parler de manière audible (1.42). E clôture cet échange par une valorisation affirmée du bricolage de A («il est TRES TRES beau », 1.43-44). Pendant ce temps, la stagiaire est clairement focalisée vers les enfants autour de la table, d'abord vers D (1.30-43), mais également vers d'autres enfants (1.44). Comme la capture d'écran le fait ressortir, la répartition en deux foyers interactionnels distincts se marque également par le positionnement dans l'espace des participants, et l'orientation des regards :

Extrait 5 : Scission de la participation.

Extract 5: Participation split

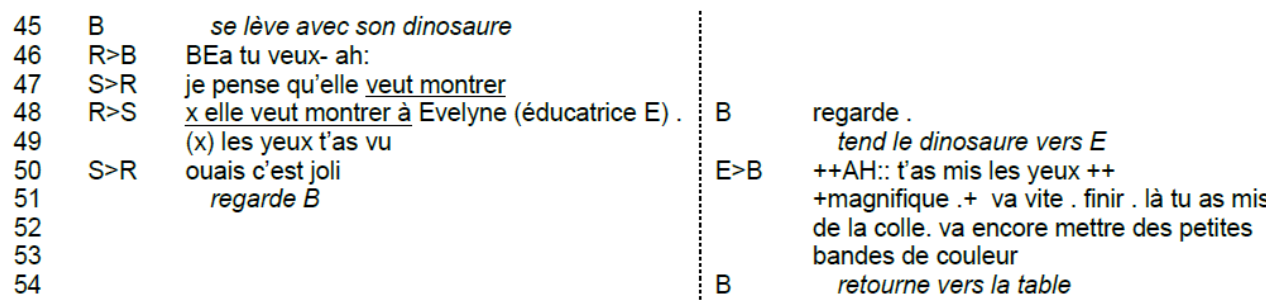

41 La valorisation manifeste du bricolage de A et l'interaction entre A et $\mathrm{E}$ attirent l'attention de certains des enfants. Ainsi, deux enfants vont montrer successivement leur bricolage à l'éducatrice E. Dans l'extrait suivant, le premier enfant (B) se lève pour montrer son dinosaure à $\mathrm{E}$ : 


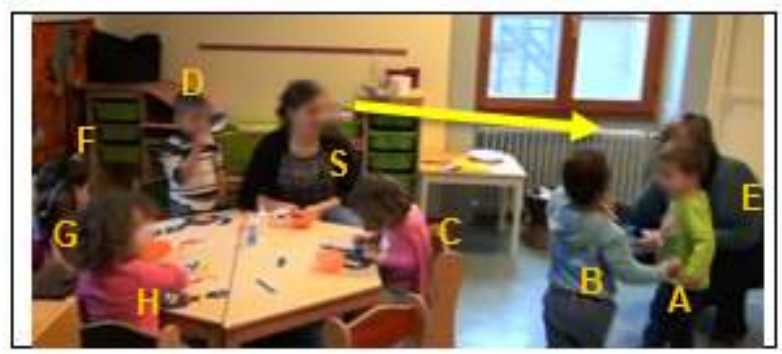

\begin{abstract}
L'énoncé de la référente est tronqué (1.46) et complété par la stagiaire («je pense qu'elle veut montrer », 1.47). La stagiaire rend manifeste qu'elle a observé la conduite de $B$, qu'elle lui a attribué une signification et qu'elle la légitime. De cette manière, elle se positionne comme responsable de l'activité en cours qui valide le déplacement de l'enfant, tout en gardant une vision d'ensemble de ce qui se passe. L'orientation de son regard (voir capture d'écran ci-dessous) rend visible que la stagiaire a élargi son champ d'attention. La référente professionnelle reprend la formulation de l'énoncé de la stagiaire en chevauchement (1.48). La référente et la stagiaire parlent ensuite des " yeux » que B a collés sur son bricolage (1. 48-50). Les deux professionnelles attribuent ainsi une signification au mouvement de $B$ et le légitiment.
\end{abstract}

Pendant ce temps, B s'approche de E et lui tend son dinosaure, en énonçant « regarde » (1. 48). Par la prosodie de ces énoncés, E valorise le dinosaure de B en le désignant de «magnifique » (1.51) et en relevant qu'elle a mis les « yeux » du dinosaure (1.50). Elle enchaîne rapidement pour inciter B de "vite finir " (1.51) et de retourner de coller «des petites bandes de couleur» (1.52-53). Ceci forme un contraste par rapport à l'interaction avec A. Comme A n'était pas occupé par le bricolage, E poursuit l'échange (extrait 5), tandis qu'elle clôt assez rapidement l'interaction avec B (extrait 6).

Dans cet extrait, l'éducatrice $E$ et la stagiaire s'engagent dans un travail de figuration. Les conduites interactionnelles de la stagiaire montrent qu'elle continue de se positionner comme responsable de l'activité en cours. Le déplacement de l'enfant B est légitimé par elle et ne constitue ainsi pas une menace pour sa face. De même, l'éducatrice $\mathrm{E}$ rend manifeste qu'elle cherche à éviter d'empiéter sur l'activité de bricolage et à menacer le positionnement de la stagiaire, en abrégeant l'interaction avec B.

Extrait 6 : Le déplacement de $B$.

Extract 6: The movement of $B$

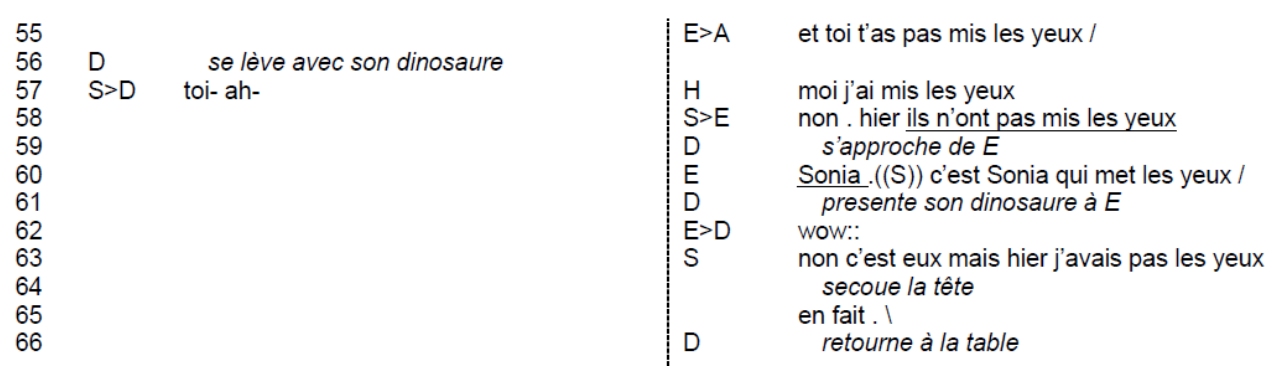

La capture d'écran rend visible que le déplacement de B attire l'attention de plusieurs enfants. Même les enfants qui continuent leur bricolage (C, F, G, D, H) observent à 
certains moments l'interaction avec B et s'engagent dans une multiactivité. L'activité de bricolage est largement suspendue. Les modalités de participation s'orientent vers l'interaction focalisée sur B. L'extrait suivant montre que le déplacement de B sera suivi par celui de D :

Extrait 7

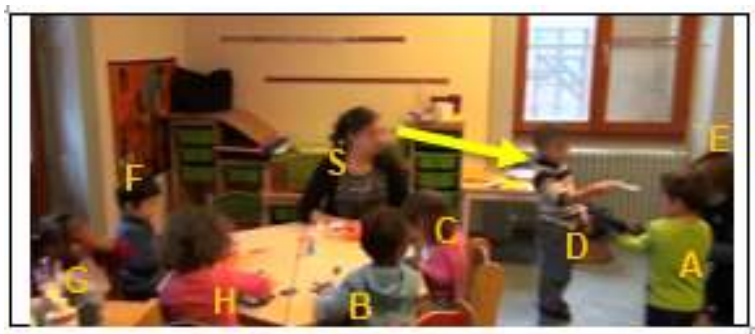

Lorsque $\mathrm{B}$ retourne à sa place, l'éducatrice $\mathrm{E}$ s'adresse à $\mathrm{A}$ et lui demande : « et toi t'as pas mis les yeux » (1.55). Lors de ces différents extraits, E cherche toujours à attribuer une signification aux conduites de $\mathrm{A}$. Au début, elle considère que A l'interpelle parce qu'il n'a pas de place à table (extrait 2), ou parce qu'il a déjà fini et cherche à valoriser le bricolage effectué (extraits 3 et 4). Elle observe ensuite que A n'a pas collé les yeux comme le font les enfants présents ce jour-là (extraits 6 et 7). Cette thématique des yeux est reprise par $\mathrm{H}$, une enfant qui est restée assise à table (« moi j'ai mis les yeux », 1. 57).

Lors du déplacement de $\mathrm{D}$, la stagiaire initie un énoncé qui reste tronqué (1.57). Elle montre qu'elle a observé que $\mathrm{D}$ s'apprête à se déplacer. Contrairement à l'extrait précédent, elle ne légitime pas explicitement ce déplacement et laisse son énoncé en suspens. La stagiaire enchaîne pour s'adresser à l'éducatrice $\mathrm{E}$ et s'aligne sur la thématique des yeux. Elle explicite que la veille, les enfants "n'ont pas mis les yeux » (1. 58). Elle répond ainsi à un énoncé adressé à $\mathrm{A}$ et rend manifeste qu'elle considère que l'éducatrice, à travers cet énoncé, s'est également adressée indirectement à elle. À travers ces conduites, la stagiaire se positionne toujours comme responsable de l'activité de bricolage en cours, mais en argumentant et en justifiant ce qui se passe (1. 58).

Dans un chevauchement des énoncés, $\mathrm{E}$ s'adresse simultanément à A pour lui demander si c'est la stagiaire qui colle les yeux («Sonia . c'est Sonia qui met les yeux / », 1.60). E valorise à nouveau le bricolage effectué par D (« wow:: », 1. 62), de façon rapide et moins accentuée qu'auparavant dans l'interaction avec B. Sans s'aligner sur l'interaction entre $\mathrm{D}$ et $\mathrm{E}$, la stagiaire s'adresse à nouveau à $\mathrm{E}$ et explique la différence dans la démarche de bricolage entre la veille et l'activité en cours («mais hier j'avais pas les yeux en fait », 1.63-65). Lors de cet extrait, la stagiaire interagit directement avec E, tandis que l'éducatrice continue à s'adresser indirectement à elle, en parlant avec les enfants.

Les conduites interactionnelles de la stagiaire, justifiant ses pratiques, semblent montrer qu'elle considère que l'éducatrice $\mathrm{E}$ met en question ce qui se passe, à travers des adressages indirects. L'éducatrice, quant à elle, rend visible qu'elle ne se focalise pas sur les pratiques de la stagiaire, mais qu'elle s'intéresse aux conduites des enfants. 
Extrait $7:$ Le déplacement de $D$.

Extract 7: : The movement of $D$

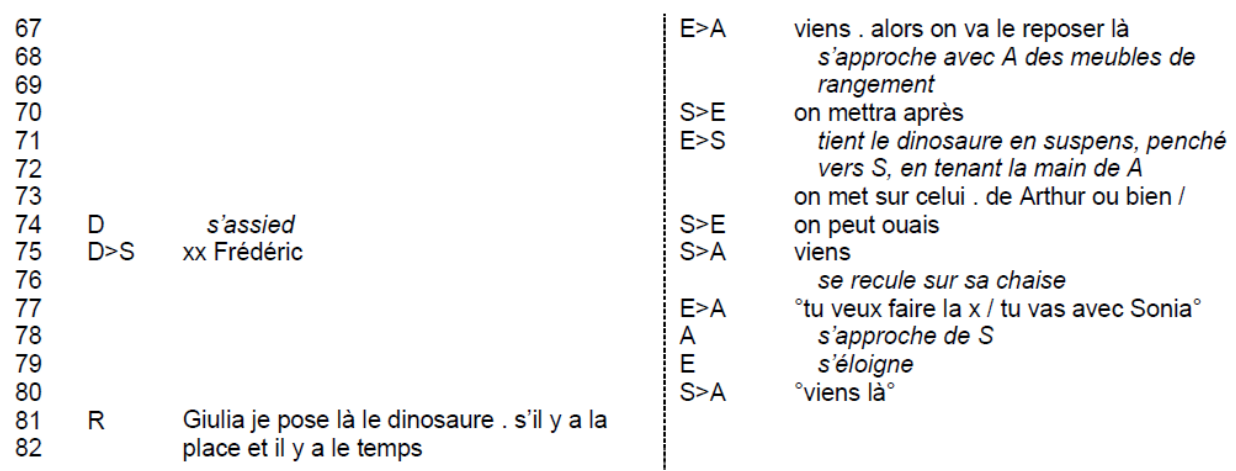

50 La discussion concernant les «yeux" du dinosaure et le déplacement de D sont observés par l'ensemble des participants. Certains enfants continuent leur bricolage (G, F) tout en observant ce qui se passe. La focalisation s'est déplacée de l'activité de bricolage sur la problématique de A qui n'a pas collé les yeux du dinosaure. Lorsque D retourne vers la table, les enfants s'orientent à nouveau vers leur activité de bricolage. E propose à A de ranger son dinosaure et s'approche ainsi de la stagiaire :

\section{Extrait 8}

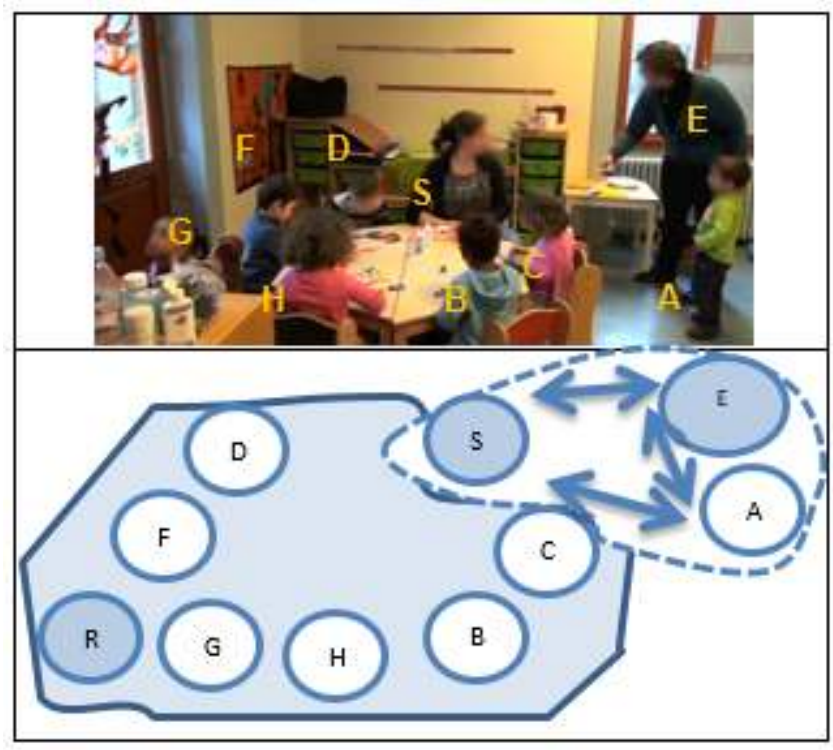

51 Pendant que l'activité collective reprend, l'éducatrice E, la stagiaire et A s'engagent dans un foyer interactionnel en aparté. E s'adresse à $\mathrm{A}$ et lui propose de "reposer » le dinosaure (1.67). Dans son énoncé, E introduit le pronom «on» qui souligne l'inscription des conduites de A dans la réalisation d'une activité collective. E et A s'approchent du meuble placé derrière la stagiaire. La stagiaire s'adresse à $E$ en énonçant «on mettra après» (1.70). L'éducatrice s'adresse alors directement à la stagiaire. Elle tient A par la main, se penche vers la stagiaire et formule une question : « on met sur celui. de Arthur ou bien/» (1. 73). La stagiaire ratifie cet énoncé par « on peut ouais » (1.74). L'utilisation du pronom « on » est initiée par E, ratifiée et reprise par la stagiaire. De façon conjointe, les deux professionnelles font ici émerger les règles de l'activité collective en cours. S'agit-il d'inclure tous les enfants dans l'activité en 
cours ou la participation de certains enfants peut-elle être différée? De façon simultanée, l'utilisation du pronom «on " positionne $\mathrm{E}$ en tant que porte-parole de A. Par l'alignement de la stagiaire, l'utilisation du pronom « on » constitue également la collectivité de l'institution, et des règles de participation qui s'y élaborent.

Ces différents éléments rendent visibles que l'éducatrice, la stagiaire et A s'engagent ici dans une négociation portant sur les modalités de l'activité en cours. Dans les extraits précédents, les interactions des différents participants ont fait émerger différentes hypothèses portant sur la signification des conduites de A.L'éducatrice E rend manifeste qu'elle s'interroge sur la signification de déplacement de A vers elle. L'enfant en question veut-il attirer son attention sur le fait qu'il n'a pas « de place à table »? Par les interventions successives de la référente, de la stagiaire, des enfants et de l'éducatrice, d'autres attributions de significations se dessinent. L'enfant A veut peutêtre montrer le bricolage qu'il a effectué pour être valorisé. Ou il se peut qu'il veuille faire comprendre qu'il n'a pas pu finir son bricolage et qu'il n'a pas collé les yeux du dinosaure. Ces attributions de significations aux conduites de A et les énoncés adressés aux enfants par l'éducatrice $\mathrm{E}$, qui constituent autant d'adressages indirects à l'intention de la stagiaire, amènent la stagiaire à annoncer qu'elle collera les yeux du dinosaure de A ultérieurement avec lui : « on mettra après » (ligne 70).

L'éducatrice ne s'aligne pas sur la projection d'une activité future, énoncée par la stagiaire. Pour la première fois dans l'ensemble des extraits de la séquence analysée, l'éducatrice s'adresse alors directement à la stagiaire. Par son intervention, elle propose une réorientation de l'activité actuelle de la stagiaire. Une telle conduite interactionnelle peut être considérée comme une menace pour la «face» de la stagiaire, de son image de soi (Goffman, 1974). Afin de moduler un tel aspect de conduite menaçante envers la face de la stagiaire, l'éducatrice mobilise de nombreuses ressources interactionnelles. Le positionnement de l'éducatrice, en proximité de la stagiaire et légèrement éloigné de la table des enfants, marque un aparté dans le déroulement des interactions. L'intonation de son énoncé est clairement interrogative, l'utilisation du prénom de l'enfant, le geste de montrer le dinosaure de A et la posture inclinée rendent visibles que l'éducatrice parle pour et à la place de l'enfant. L'aspect éducatif est mis en avant de la scène interactionnelle. L'éducatrice se positionne ainsi comme porte-parole de l'enfant, et non comme professionnelle experte qui donne une consigne à une stagiaire. Cet extrait fait ressortir une certaine « déconstruction » d'un positionnement de locuteur (Goodwin, 2007, p. 26), lorsque l'éducatrice E se constitue comme animateur ou comme « sounding box » (op.cit. p. 26) pour la position de A.

Dans cette situation, A n'a pas les ressources nécessaires pour donner des indices clairs de ce qu'il cherche à exprimer. La signification des conduites de A est élaborée de façon collective. A parle peu et de façon presque inaudible. Son déplacement et la monstration de son dinosaure vont initier un processus d'enquête collective pour attribuer une signification à ses conduites. Les énoncés de l'éducatrice $\mathrm{E}$, de la référente et de la stagiaire, mais aussi les déplacements des enfants B et D, ainsi que l'observation des éléments matériels présents (les «yeux» du dinosaure) construisent progressivement une compréhension de l'ensemble des participants de ce qui est en train de se passer. La position de locuteur de A est prise en charge par l'engagement collectif des interactants, et notamment par l'éducatrice $\mathrm{E}$.

Après cette parenthèse de négociation entre l'éducatrice et la stagiaire, celle-ci invite $\mathrm{A}$ à la rejoindre («viens », 1. 75). L'éducatrice encourage A à aller avec la stagiaire (1. 77), 
avant de s'éloigner et de passer dans une autre salle. La prise en compte de A par la stagiaire, et l'activité mise en place pour coller des "yeux»sur son dinosaure constituent ainsi des indices de clôture de cette séquence et sont suivies par le départ de l'éducatrice. Les enfants ne s'orientent pas vers l'interaction entre $\mathrm{E}, \mathrm{S}$ et $\mathrm{A}$ et la référente professionnelle n'y intervient pas non plus. Elle continue son découpage et s'adresse à un enfant pour lui indiquer qu'elle pourra éventuellement faire son bricolage (1. 81-82). Les deux foyers interactionnels se répartissent comme suit :

Extrait 8 : Interaction entre $\mathrm{E}, \mathrm{S}$ et $\mathrm{A}$.

Extract 8: Interactions between $E, S$ and $A$

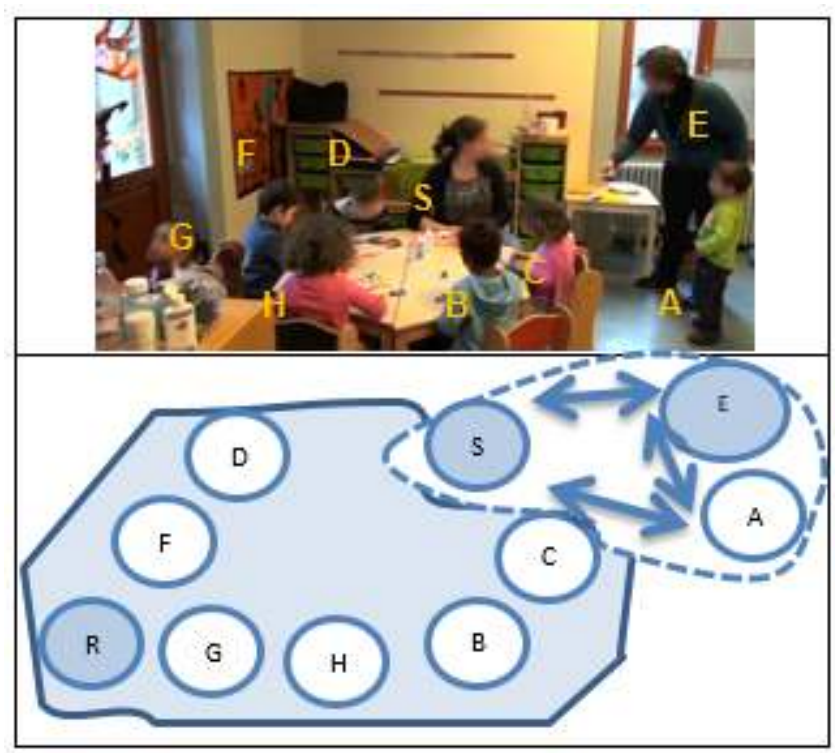

Après le départ de l'éducatrice, la stagiaire interagit avec $\mathrm{A}$ et s'oriente à nouveau vers l'activité de bricolage menée collectivement avec le groupe des enfants : 


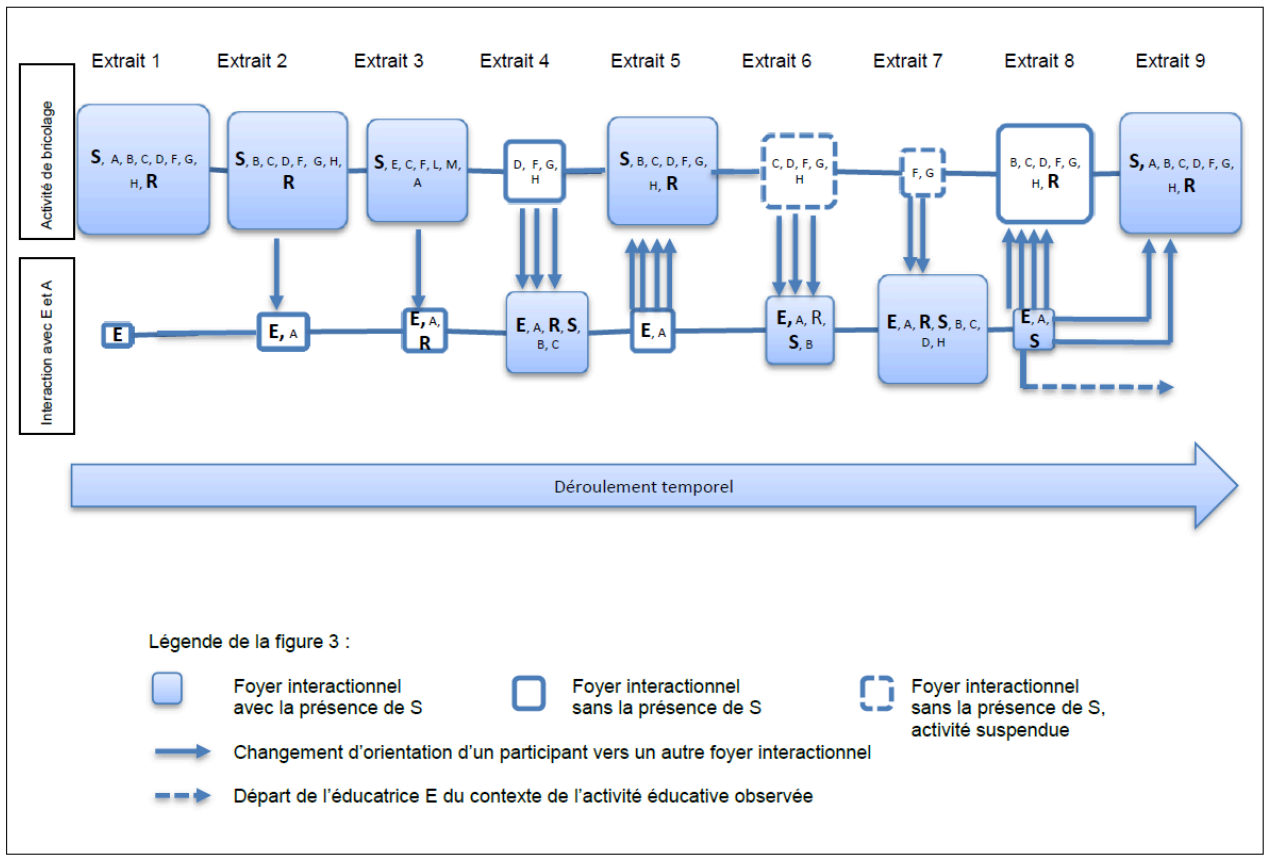

Pendant cet extrait, la stagiaire accompagne A dans le collage d'une perle sur son bricolage. Chacun des enfants se focalise sur la réalisation de son bricolage et la référente commence à ranger des papiers.

\section{L'engagement de la stagiaire à travers des modalités de participation fluctuantes}

L'analyse des différents extraits montre que les enfants et les professionnelles présents dans le contexte interactionnel s'engagent de multiples façons dans la configuration de participation située. Le nombre d'interactants focalisés sur l'activité de bricolage ou sur l'interaction concernant A varie dans le déroulement interactionnel. Ces changements peuvent se résumer schématiquement comme suit :

Figure 3 : Changements dans la configuration de participation située.

Figure 3: Changes in the participatory configuration

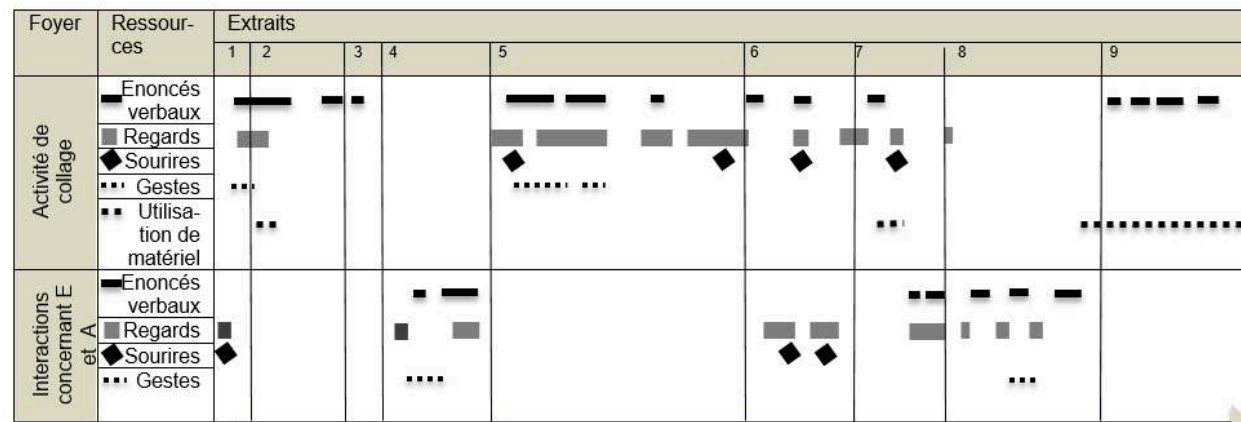

Déroulement temporel 
59 L'analyse des données empiriques éclaire la complexité des interactions avec de multiples participants. Le schéma représente les orientations de chacun des participants vers un des foyers interactionnels en distinguant les professionnels (en gras : Stagiaire (S), Référente (R), Éducatrice (E)) ainsi que les enfants (sans gras : A, B, $\mathrm{C}, \mathrm{D}, \mathrm{F}, \mathrm{G}, \mathrm{H}$ ). Il montre également les modifications des engagements de la stagiaire qui s'oriente principalement vers l'activité de bricolage dans les extraits 1,2,3,5, et 9, et vers l'interaction focalisée sur A dans les extraits 4, 6, 7 et 8. Les changements d'orientation des différents participants sont indiqués par des flèches. Le nombre de participants qui se réorientent est schématisé par le nombre de flèches correspondantes.

60 Les changements dans la configuration de participation observée sont fréquents. Dans de nombreux contextes professionnels de l'éducation, du travail social ou de la santé, le déroulement des interactions et des cours d'action ne suit pas une procédure stabilisée et préétablie. En ce qui concerne l'éducation de la petite enfance, notamment, les pratiques professionnelles s'accomplissent dans des situations marquées par une forte imprévisibilité (Filliettaz \& Zogmal, à paraître). Lors de l'activité de bricolage étudiée, les modalités de participation varient en fonction du nombre des activités en cours et du nombre des foyers interactionnels présents.

61 Les changements dans la configuration de participation font ressortir que l'arrivée de l'éducatrice $\mathrm{E}$ amène une perturbation de l'activité de bricolage. Cette perturbation se situe d'abord en parallèle à une activité de bricolage qui peut être désignée comme activité principale (extraits 1 à 3). Ensuite, l'activité de bricolage est mise en suspens pour une partie des interactants qui s'orientent vers l'interaction entre l'éducatrice et A (extrait 4), avant de reprendre l'activité principale (extrait5). Une telle hiérarchisation entre activité principale et secondaire se modifie cependant dans les extraits 6 et 7. Les déplacements de deux des enfants de la table du bricolage vers l'éducatrice $\mathrm{E}$ attirent une focalisation de la plupart des participants vers les interactions avec l'éducatrice. Les échanges concernant les yeux collés ou non sur le dinosaure se déroulent ici de manière compétitive avec la poursuite de l'activité de bricolage. Ce basculement de la focalisation des modalités de participation sera suivi par une sorte de parenthèse de négociation entre la stagiaire, l'éducatrice et $\mathrm{A}$. Comme l'analyse empirique le montre, cette négociation va amener la stagiaire à réorienter son activité. L'activité collective du bricolage reprend dans le dernier extrait, en y incluant A.

62 Ces changements émergeants des modalités de participation dans les différents foyers interactionnels et les diverses activités en cours donnent lieu à des engagements diversifiés de la part des interactants. Une centration sur les conduites interactionnelles mobilisées par la stagiaire permet de s'intéresser à la problématique de la participation de la stagiaire dans un contexte avec de multiples participants. Dans les données empiriques étudiées, l'engagement de la stagiaire ( $\mathrm{S}$ ) varie et prend des formes multiples. Elle mobilise un large éventail de ressources multimodales de façon imbriquée. Afin de pouvoir analyser l'orientation et la focalisation de ces différentes ressources et pour les retracer dans leurs dimensions de séquentialité et de simultanéité, le schéma suivant différencie les verbalisations, les orientations des regards vers les différents participants à l'interaction, les sourires, les gestes et l'utilisation du matériel : 
Figure 4 : Déploiement des ressources multimodales par la stagiaire dans un contexte multiparticipants.

Figure 4: Deployment of multimodal resources by the trainee in a context with multiple participants

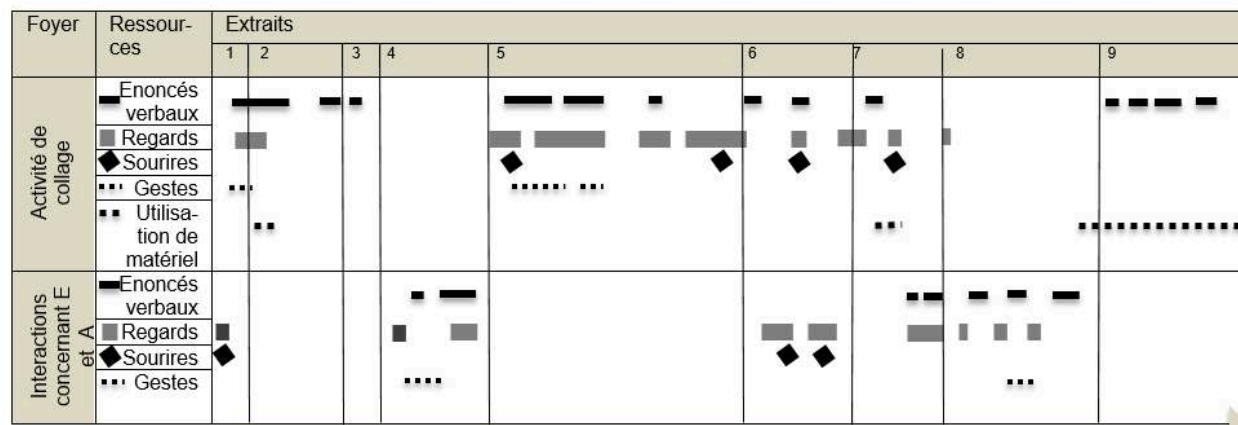

Déroulement temporel

Le premier extrait présenté (Extrait 1) montre que la stagiaire se focalise sur le bricolage collectif en cours, tout en observant le contexte institutionnel plus large et l'arrivée de l'éducatrice E. L'arrivée de l'éducatrice peut ici être considérée comme une possible "induction» d'une scission de la participation. La stagiaire s'y oriente et s'engage ainsi dans une multiactivité. La scission de la participation se met en place dans l'extrait 2, à travers le déplacement de A vers l'éducatrice, et la poursuite de l'activité de bricolage par la stagiaire et les autres enfants. En ce qui concerne la stagiaire, elle s'oriente vers un seul des foyers interactionnels dans cet extrait 2. Elle reste focalisée sur l'activité de collage avec les enfants, observe les conduites de plusieurs enfants et rend ses observations publiquement manifestes. De cette manière, elle s'engage dans un seul foyer interactionnel à travers l'accomplissement d'une multiactivité.

4 Dans les trois extraits suivants, la focalisation de la stagiaire oscille, mais elle reste toujours orientée vers un seul foyer interactionnel à la fois. En premier, la stagiaire continue à s'orienter vers le foyer interactionnel centré sur l'activité de bricolage (Extrait 3), avant de s'orienter vers l'éducatrice E (extrait 4), et de se focaliser à nouveau sur les enfants autour de la table de bricolage (extrait 5). Les oscillations de la focalisation se font dans la séquentialité du déroulement interactionnel et la stagiaire s'engage successivement dans une activité dans un seul des foyers interactionnels existants.

Lors des extraits 6 et 7, la stagiaire s'engage simultanément dans deux foyers interactionnels à travers une multiactivité. Elle continue à observer les enfants qui bricolent, à interagir avec la référente, tout en observant les interactions avec l'éducatrice $\mathrm{E}$ et en interagissant avec elle. Cet engagement dans une multiactivité déployée vers deux foyers interactionnels donne lieu à une focalisation sur un seul des foyers interactionnels existants dans l'extrait 8. Dans une sorte de parenthèse de négociation, la stagiaire réoriente le cours de son activité afin de permettre à $\mathrm{A}$ de coller des "yeux " sur son dinosaure. Le dernier extrait montre qu'une interaction collective focalisée se met en place lors de la clôture de la séquence analysée.

Pour étudier ces phénomènes d'engagement d'un des participants à une situation d'interaction avec de multiples participants, il est intéressant de considérer les types possibles de l'engagement dans différentes modalités de participation comme suit : 
Tableau 2 : Types d'engagement d'un interactant dans des modalités de participation diverses. Table 2: Types of engagement of an interactant in various participatory modes

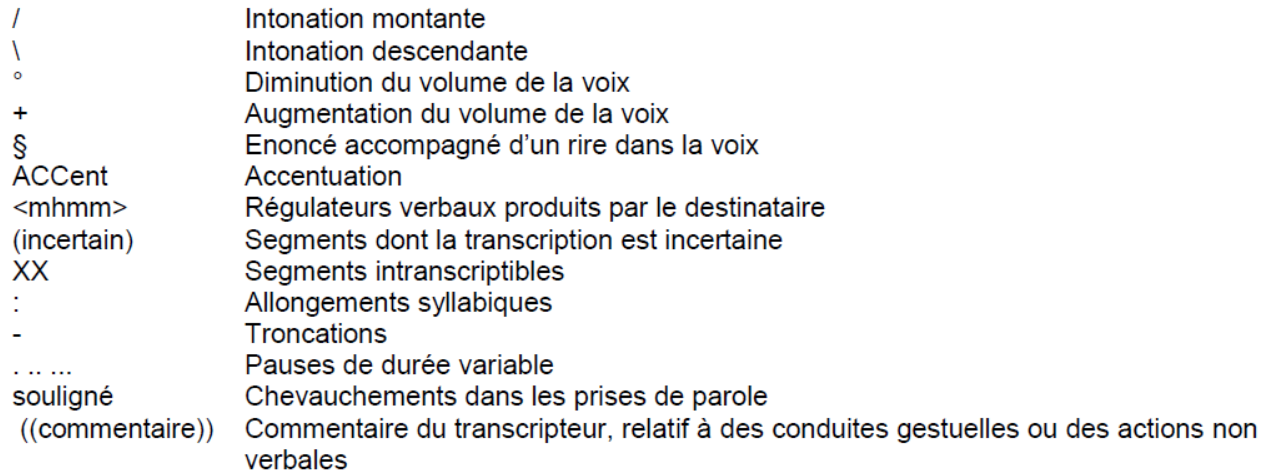

Dans certains moments des extraits analysés, la stagiaire rend visible qu'elle se focalise sur un seul foyer interactionnel. Elle se centre sur l'activité de bricolage, de façon manifeste (extraits 2, 3, 5 et 9) ou se focalise sur l'interaction concernant l'éducatrice $\mathrm{E}$ (extraits 4 et 8 ). Dans tous ces extraits, à part celui de l'extrait B, il s'agit d'un engagement dans un seul des foyers interactionnels existants à travers une seule activité (type b). De cette manière, la stagiaire s'engage principalement par l'accomplissement d'activités focalisées qui se succèdent dans un "agencement temporel » (Mondada, 2017, p. 335) de l'interaction. En ce qui concerne l'extrait 2, la stagiaire se focalise sur l'activité de bricolage et donc sur un seul des foyers interactionnels existants. Cependant, elle s'engage dans une multiactivité focalisée (type $\left(\mathrm{d}^{1}\right)$, en interagissant avec plusieurs enfants de façon simultanée.

Comme déjà évoqué, lors de certains moments, la stagiaire se focalise sur les deux foyers interactionnels de façon simultanée et s'engage ainsi dans une multiactivité polyfocalisée de type $\mathrm{d}^{2}$ (extraits 1, 6, et 7). Le déroulement interactionnel est alors marqué par une série de « simultanéités organisées séquentiellement »(Mondada, 2017, p. 334).

69 Ces différentes modalités de participation déployées par la stagiaire se succèdent rapidement. Elles impliquent que la stagiaire varie ses formes d'adressages et ses focalisations. Elles amènent la stagiaire à s'engager dans des activités différentes, orientées vers un accompagnement des enfants, vers l'attribution d'une signification aux conduites de A, vers une explicitation de son activité et de ses modalités ou vers une négociation portant sur les pratiques considérées comme légitimes.

\section{Conclusion : La participation des novices et ses enjeux pour la formation en situation de travail}

70 Se positionner dans un contexte de stage dans l'éducation de l'enfance mobilise des compétences diverses. Dans les données empiriques analysées, la stagiaire mène une activité de bricolage avec un groupe d'enfants. Cela implique de préparer du matériel, d'aménager l'espace, de s'installer, d'interagir avec les enfants pour réguler leurs conduites et d'assurer la progressivité de l'activité en cours. Les conduites de la référente professionnelle rendent visible que c'est la stagiaire qui est censée assurer la responsabilité de la conduite de l'activité et de l'accompagnement du groupe d'enfants. Par son recul spatial à l'égard de l'activité en cours, par sa centration sur un soutien 
matériel et par ses interventions très succinctes, la référente professionnelle se met manifestement dans un positionnement de retrait. Pour la stagiaire, il s'agit de « savoir gérer ».

71 Par ailleurs, le contexte de stage implique que la stagiaire collabore et s'ajuste au collectif de travail. Une coordination entre la référente et la stagiaire s'est mise en place tout au long de la réalisation de l'activité de bricolage. Pour la stagiaire, l'arrivée de l'éducatrice $\mathrm{E}$ amène une nouvelle nécessité de s'ajuster. Cela fait ressortir une tension inhérente à une telle situation. Savoir assurer l'accomplissement du bricolage et mener à terme une activité projetée demande d'orienter les conduites des enfants présents et d'adopter une position d'animatrice responsable. Savoir collaborer, s'ajuster aux membres de l'équipe et accepter des réorientations de son activité nécessite de se positionner en tant que stagiaire en formation.

La participation à un contexte avec de multiples participants demande ainsi de savoir varier les positionnements diversifiés. Ces variations peuvent survenir dans une successivité, en formant des parenthèses ou des apartés. Par ailleurs, elles émergent également de façon simultanée dans le déroulement des interactions. L'engagement dans deux foyers interactionnels par une multiactivité polyfocalisée (extraits 7 et 8) offre des ressources interactionnelles pour un positionnement orienté simultanément vers les dimensions éducative et formative du contexte. Dans la séquence analysée, l'arrivée de l'éducatrice et ses interventions «dérangent » l'activité de la stagiaire et constituent une "rupture» (Pastré et al., 2006) dans ce qui se passe. L'analyse des données montre que l'éducatrice rend visible qu'elle intervient pour et à la place de A. L'éducatrice mène une forme d'enquête pour attribuer une signification aux conduites de $\mathrm{A}$ et elle établit ainsi une monstration d'une pratique professionnelle experte. Les ressources interactionnelles mobilisées par l'éducatrice s'inscrivent dès lors dans une dimension éducative orientée vers les enfants. L'éducatrice intervientelle également pour former la stagiaire, pour lui faire « voir» ce qui est pertinent dans la situation donnée et pour lui montrer les éléments pertinents à prendre en compte ? De tels ingrédients d'un tutorat distribué ne sont pas rendus manifestes. Une éventuelle dimension formative disparaît derrière la dimension éducative.

73 C'est le soin donné de ne pas se positionner comme experte, c'est l'absence d'une posture rendue manifeste de tutrice, qui préserve la «face» de la stagiaire et lui offre ainsi une opportunité de réorienter son activité sans se sentir désavouée en présence des enfants. L'éducatrice, en procédant par des adressages indirects, en s'alignant aux conduites des enfants qui font émerger de nouvelles attributions de significations, s'appuie sur les phénomènes d'interdépendance inhérents aux interactions. Ces procédés permettent à la stagiaire d'identifier l'action à mettre en place pour répondre aux conduites de A, comme son énoncé «on mettra plus tard» (ligne 70) le montre. L'intervention directe de l'éducatrice porte ensuite uniquement sur le moment où $\mathrm{A}$ peut coller les yeux sur le dinosaure. La parenthèse de négociation rendue publiquement manifeste peut ainsi rester courte et s'accomplir à travers deux, trois énoncés succincts. L'occultation d'une posture de tutrice est ici une méthode, pour ne pas dire une "ethnométhode» (Garfinkel, 2007), qui permet de réorienter l'activité effectuée par la stagiaire, sans lui faire perdre sa " face ».

Une telle intervention largement indirecte et l'occultation de la dimension formative forment une opportunité d'apprentissage. Pour s'en saisir, la stagiaire est amenée à faire preuve d'une grande palette de compétences interactionnelles. $\mathrm{Vu}$ 
l'imprévisibilité du déroulement interactionnel, elle doit continuer à "garder la main " sur son activité, malgré les interventions des autres professionnelles et malgré les déplacements réitérés des enfants qui "désertent " la table de bricolage. L'analyse interactionnelle montre que la stagiaire s'ajuste aux conduites des autres interactants, qu'elle les légitime et qu'elle les intègre dans son activité. De cette façon, elle ne subit pas l'émergence des conduites des autres participants comme une menace pour sa propre activité, mais s'appuie sur ce qui advient pour élargir sa compréhension de ce qui passe et pour réorienter ses pratiques. Ainsi, la stagiaire ne se focalise pas sur sa propre activité, mais s'oriente vers l'accomplissement des activités collectives. Elle sait " lâcher prise " lorsque les enfants quittent temporairement l'activité de bricolage, en vue de maintenir et de reprendre la progressivité de l'activité en cours. Par ailleurs, la stagiaire s'engage dans un important travail de figuration.

La stagiaire rend visible, tout au long de la séquence étudiée, qu'elle sait maintenir son engagement dans la configuration de participation située. Elle observe ce qui se passe et montre ce qu'elle comprend des conduites des enfants lorsqu'ils se déplacent. Les sourires fréquents de la stagiaire, adressés à un enfant, à un autre, à l'éducatrice ou à la référente professionnelle semblent simultanément exprimer une légitimation des conduites des enfants, et un alignement aux interventions des professionnelles présentes. Ses sourires rendent manifeste qu'elle garde la maîtrise de son activité et peut préserver sa face.

76 La stagiaire sait « voir » ce qui est pertinent dans un contexte donné, elle sait varier ses focalisations, tenir compte d'un enfant individuellement et de l'ensemble du groupe d'enfants. Elle sait également "voir " ce que les professionnelles attendent d'elle, et s'inscrire dans les attentes normatives de l'équipe éducatrice. Par ailleurs, la stagiaire ne maintient pas uniquement sa propre participation à l'activité en cours, elle aménage des opportunités de participation pour les enfants. Dans les métiers orientés vers la prise en charge d'autrui, la participation à une communauté de pratique ne nécessite pas uniquement d'assurer sa propre participation. Simultanément, il s'agit de veiller à aménager des opportunités de participation pour les bénéficiaires de la prise en charge. La participation de chacun se construit collectivement, à travers les interactions entre la stagiaire, l'éducatrice, les enfants et la référente professionnelle.

L'analyse menée montre la complexité de la notion de participation qui s'inscrit dans des dimensions pragmatiques, relationnelles, épistémiques et dynamiques des interactions avec de multiples participants. Sur un plan pragmatique, savoir participer à des configurations interactionnelles implique de construire des définitions partagées de la situation, ainsi que l'accomplissement d'activités collectives. La participation mobilise des positionnements relationnels, à travers les jeux d'adressages et de figuration. La dimension épistémique s'accomplit par la construction de significations partagées et par leur mise en visibilité par les différents participants à l'interaction. Tous ces différents aspects s'inscrivent dans un déroulement dynamique, ordonné de façon séquentielle et simultanée.

78 En quoi la compréhension des interactions avec de multiples participants peut-elle constituer un apport pour les problématiques de la formation en situation de travail ? En premier lieu, une analyse fine des interactions permet de mieux comprendre les pratiques de formation réelles, accomplies en situation de travail. Elle fait notamment ressortir l'imbrication des dimensions productives et constructives, déjà identifiées par les tenants de la didactique professionnelle (Samurcay \& Rabardel, 2004). L'analyse 
montre que dans les métiers de la prise en charge d'autrui, la dimension de formation peut rester peu visible et même occultée à certains moments. Par ailleurs, il s'avère que les formes complexes de participation permettent d'accomplir plusieurs actions à la fois et offrent ainsi des ressources pour concilier les objectifs formatifs et les exigences du travail. Pour l'éducatrice, l'enjeu est double et s'inscrit dans une tension. D'une part, elle cherche à intervenir sans faire perdre la face à la stagiaire. D'autre part, il s'agit de faire comprendre à celle-ci la nature formative de son intervention. L'occultation d'un positionnement d'experte va ainsi de pair avec la mise en visibilité des pratiques éducatives considérées comme pertinentes. Les interactions avec l'enfant offrent des occasions pour s'adresser indirectement à la stagiaire. Les phénomènes de scission de la participation permettent à l'éducatrice d'accompagner la stagiaire dans l'accomplissement de son travail, tout en aménageant une opportunité d'apprentissage. En miroir, la stagiaire parvient simultanément à maintenir l'animation de l'activité de travail ainsi que l'engagement dans une démarche formative, à travers l'émergence des différentes formes de participation.

Des analyses interactionnelles peuvent enrichir des dispositifs de la formation initiale, ainsi que des formations continues, orientées vers les tuteurs, comme les praticiensformateurs ou les référents professionnels. Dans le cadre de la formation initiale des stagiaires observées, le stage de troisième année est désigné comme un "stage en responsabilité et maîtrise de situations professionnelles ». L'objectif consiste ainsi à "maîtriser» l'animation des activités éducatives. La terminologie utilisée dans le contexte de la formation initiale semble signifier qu'il s'agit de savoir " gérer » de façon autonome, de savoir «faire seul». La notion de «maîtrise» met l'accent sur une activité individuelle des apprenants. Des démarches d'analyses interactionnelles peuvent montrer que la conduite d'activités éducatives s'accomplit à travers des activités collectives. La tension entre le maintien de la progressivité de l'activité et des réorientations et des ajustements aux imprévus ne concerne pas uniquement la stagiaire, mais l'ensemble des participants à l'interaction. De nombreux dispositifs de formation initiale et continue sont orientés fortement sur les pratiques professionnelles accomplies individuellement. Un élargissement sur la dimension collective pourrait donner des clefs de compréhension pertinentes pour se positionner dans les situations de travail et de formation. Des démarches d'analyse des interactions, basées sur des films vidéo réalisés par les éducatrices et éducateurs en formation, pourraient faire ressortir les complexités des situations d'interaction avec de multiples participants. Certains projets pilotes ont pu être menés dans le cadre de dispositifs de formation continue (Durand \& Trébert, 2018; Zogmal \& Durand, 2020) et également dans la formation initiale (Filliettaz \& Trébert, 2015). Ces projets pilotes montrent que les participants à la formation s'orientent aisément vers le repérage de la dimension collective de leur activité et vers les relations d'interdépendances qui prennent place entre l'activité des stagiaires et celle des autres membres des équipes éducatives. Pour les novices, une meilleure compréhension de la complexité des interactions avec de multiples participants peut leur faciliter la planification, la réalisation et l'évaluation des activités. Une meilleure prise en compte de la dimension collective des situations éducatives leur donnerait également de nouvelles perspectives en ce qui concerne les positionnements relationnels.

80 Quant à la formation continue des tuteurs, il semble intéressant de mobiliser des démarches d'analyse des interactions afin d'éclairer les pratiques de formation peu visibles, fugitives et parfois occultées. Lors de l'accompagnement des stagiaires, une 
telle posture d'analyse permet de dépasser une focalisation sur l'action individuelle des novices, et de l'inscrire dans le contexte des activités collectives menées. Par ailleurs, une réflexion autour des dimensions implicites et explicites des situations de formation peut contribuer à une meilleure reconnaissance de l'activité des tuteurs, pour les professionnels eux-mêmes, ainsi que dans le cadre des dispositifs de formation.

\section{BIBLIOGRAPHIE}

Brown, P., \& Levinson, S.C. (1978). Politness: Some universals in language usage, 4. Cambridge : Cambridge University Press.

Durand, I. (2017). Le positionnement du stagiaire dans la relation tutorale : une analyse interactionnelle des pratiques d'aide dans la formation à l'éducation de l'enfance. Thèse de doctorat en Sciences de l'éducation, Université de Genève.

Durand, I., \& Trébert, D. (2018). Quand l'outil des chercheurs devient celui des praticiens : Un Groupe d'Analyse des Interactions en formation de formateurs. In I. Vinatier, L. Filliettaz \& M. Laforest (Eds.), L'analyse des interactions dans le travail : outil de formation professionnelle et de recherche (pp. 51-76). Dijon : Éditions Raison \& Passions.

Durand, I., Trébert, D., \& Filliettaz, L. (2015). Offre et prise de place : L'accomplissement des configurations de participation à l'interaction tutorale dans le contexte de la formation professionnelle en éducation de la petite enfance. In K. Balslev, S. Cartaut, L. Filliettaz, \& I. Vinatier (Eds.), Pratiques professionnelles en formation : la part du langage (pp. 31-60). Paris : L'Harmattan.

Egbert, M. M. (1997a). Schisming: The collaborative transformation from a single conversation to multiple conversations. Research on Language \& Social Interaction, 30(1), 1-51.

Egbert, M. (1997b). Some interactional achievements of other-initiated repair in multiperson conversation. Journal of Pragmatics, 27, 611-634.

Filliettaz, L. (2014). L'interaction langagière : un objet et une méthode d'analyse en formation d'adultes. In J. Friedrich \& J. Pita, J. (Eds.), Recherches en formation des adultes : un dialogue entre concepts et réalité (pp. 127-162). Dijon : Éditions Raison et Passions.

Filliettaz, L. (2018). Interactions verbales et recherche en éducation; Principes, méthodes et outils d'analyse. Carnets des sciences de l'éducation. Genève : Université de Genève.

Filliettaz, L., de Saint-Georges, I., \& Duc, B. (2008). « Vos mains sont intelligentes! » : Interactions en formation professionnelle initiale. Université de Genève : Cahiers de la section des sciences de l'éducation, 117.

Filliettaz, L. Rémery, V., \& Trébert, D. (2014). Relation tutorale et configurations de participation à l'interaction. Analyse de l'accompagnement des stagiaires dans le champ de la petite enfance. Activités, Association Recherches et Pratiques sur les ACTivités, 11(1), 22-46.

Filliettaz, L., \& Trébert, D. (2015). Le travail comme objet d'analyse dans les espaces interprétatifs de l'alternance : le cas de la formation ES des éducatrices et éducateurs de l'enfance. In V. Lussi 
Borer, M. Durand \& F. Yvon (Eds.), Analyse du travail et formation dans les métiers de l'éducation (pp. 161-178). Bruxelles : De Boeck.

Filliettaz, L., \& Zogmal, M. (Eds.) (à paraître). Mobiliser et développer des compétences interactionnelles en situation de travail éducatif. Toulouse : Éditions Octarès.

Garfinkel, H. (1967). Studies in Ethnomethodology. Englewood Cliffs (New Jersey): Prentice-Hall.

Garfinkel, A. (2007). Recherches en ethnométhodologie. Paris : Presses Universitaires de France.

Goffman, E. (1974). Les rites d'interaction. Paris : Éditions de Minuit.

Goffman, E. (1987). Façons de parler. Paris : Éditions de Minuit.

Goffman, E. (1991). Les cadres de l'expérience. Paris : Éditions de Minuit.

Goodwin, C. (2007). Interactive Footing. In E. Holt \& R. Clift (Eds.), Reporting Talk: Reported Speech in Interaction (pp. 16-46). Cambridge : Cambridge University Press.

Grosjean, M. (1995). Contextualisations vocales en situations de travail. Connexions, 65-1, 27-42.

Grosjean, M., \& Lacoste, M. (1999). Communication et intelligence collective. Le travail à l'hôpital. Paris : Presses universitaires de France.

Grosjean, M., \& Traverso, V. (1998). Les cadres participatifs dans les polylogues : problèmes méthodologiques. In F. Cabasino (Ed.), Du dialogue au polylogue (pp. 51-67). Rome : CISU.

Kerbrat-Orecchioni, C. (1992). Les interactions verbales [vol. 2]. Paris : Armand Colin.

Kress, G., Jewitt, C., Ogborn, J., \& Charalampos, T. (2001). Multimodal teaching and learning. The rhetorics of the science classroom. London : Continuum.

Lave, J., \& Wenger, E. (1991). Situated learning: Legitimate peripheral participation. Cambridge : Cambridge University Press.

Markaki, V., \& Filliettaz, L. (2017). « Comment faire les remarques dans l'effervescence du travail ?» : une analyse interactionnelle des scissions des cadres participatifs dans la formation professionnelle des éducateurs de la petite enfance. Travaux Neuchâtelois de Linguistique, 67, 79-102.

Mayen, P. (2002). Le rôle des autres dans le développement de l'expérience. Éducation permanente, 151, 87-107.

Mondada, L. (2006). La compétence comme dimension située et contingente, localement évaluée par les participants. Bulletin suisse de linguistique appliquée, 84, 83-119.

Mondada, L. (2017). Le défi de la multimodalité en interaction. Revue française de linguistique appliquée, XXII(2), 71-87.

Pastré, P., Mayen, P., \& Vergnaud, G. (2006). La didactique professionnelle. Revue française de pédagogie, 154, 145-198.

Sacks, H., Schegloff, E., \& Jefferson, G. (1974). A Simplest Systematics for the Organization of Turn-Taking for Conversation. Language, 50, 696-735.

Samurçay, R. \& Rabardel, P. (2004). Modèles pour l'analyse de l'activité et des compétences. In R. Samurçay \& P. Pastré (Eds.), Recherches et pratiques en didactique professionnelle (pp. 163-180). Toulouse : Octarès.

Simmel, G. (1902). The number of members as determining the sociological form of the group. II. American Journal of Sociology, 8(2), 158-196. 
Traverso, V. (2012). Ad hoc-interpreting in multilingual work meetings. In C. Baraldi \& L. Gavioli (Eds.), Coordinating participation in dialogue interpreting (pp. 149-176). Amsterdam : John Benjamins.

Traverso, V. (1997). Des échanges à la poste : dilogues, trilogues, polylogue(s) ? Cahiers de Praxématique, 28, 57-77.

Vion, R. (2000). La communication verbale. Paris : Hachette éducation.

Wenger, E. (1999). Communities of practice: Learning, meaning, and identity. Cambridge : Cambridge University Press.

Zarifian, Ph. (2001). Objectif compétence. Pour une nouvelle logique. Paris : Éditions Lia.

Zogmal, M., \& Durand, I. (à paraître). De l'observation des enfants à l'analyse interactionnelle : contributions de la recherche à la formation continue des éducateurs et éducatrices de l'enfance. In L. Filliettaz et al. (Eds.), De l'observation à l'intervention : les usages de l'analyse interactionnelle en formation. Revue phronesis.

\section{ANNEXES}

\section{Conventions de transcription}

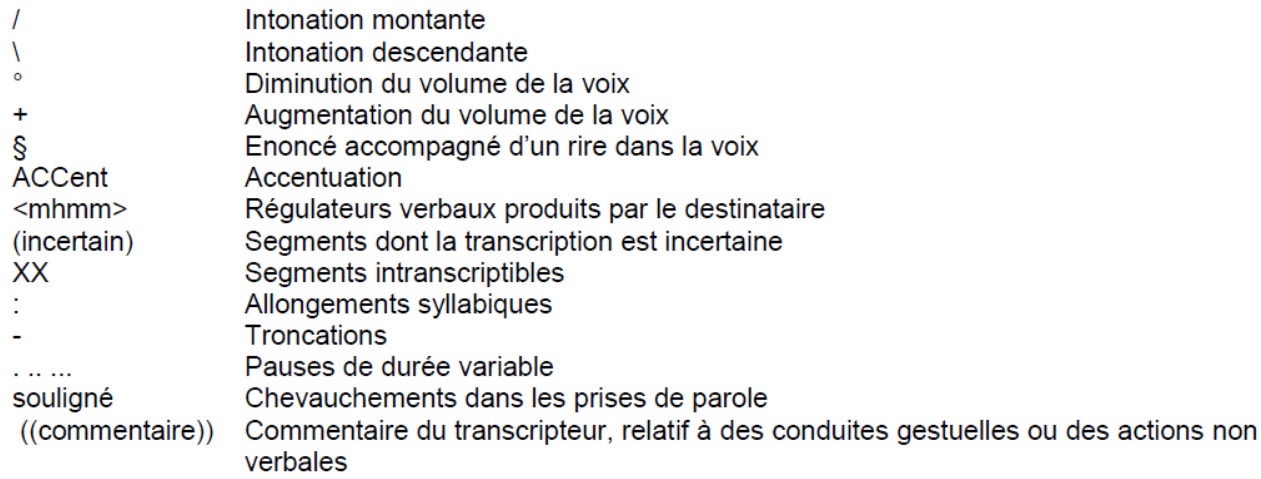

\section{NOTES}

1. Comme signe de reconnaissance pour le travail effectué par les femmes travaillant dans le domaine de la petite enfance (une très grande majorité de plus de $96 \%$ !), ce texte utilisera la forme du féminin pour désigner les professionnelles de l'enfance et souligne ainsi le manque regrettable d'éducateurs dans ce champ.

\section{RÉSUMÉS}

Dans les métiers de l'éducation et des soins, la formation professionnelle en situation de travail se déroule fréquemment dans des interactions regroupant de multiples participants. Pour les 
étudiants en formation, il s'agit dès lors d'apprendre à participer aux activités en cours en s'insérant dans une équipe de travail et en s'ajustant aux usagers. Au croisement des recherches portant sur l'apprentissage en situation de travail et des perspectives interactionnelles en analyse du travail, cet article propose une étude empirique centrée sur les modalités de participation d'une étudiante stagiaire dans le champ de l'éducation de l'enfance. Il s'intéresse en particulier à une activité éducative menée par la stagiaire, en présence des enfants et de deux éducatrices expérimentées. La notion de configuration de participation permet d'aborder comment les différents participants établissent une définition de la situation et varient leurs focalisations sur différents aspects des activités collectives en cours. L'analyse empirique des données audio-vidéo et des transcriptions permet de souligner que les positionnements relationnels s'effectuent à travers des jeux d'adressages et de figuration. Sur le plan épistémique, les interactants co-construisent des significations partagées, rendues mutuellement manifestes dans l'accomplissement des interactions. Des changements incessants s'inscrivent dans un déroulement interactionnel dynamique, organisé de façon séquentielle et simultanée. Dès lors, savoir participer à des situations de formation dans et par le travail s'inscrit dans des dimensions pragmatiques, relationnelles, épistémiques et dynamiques des interactions. L'analyse des interactions permet d'éclairer ces phénomènes et les enjeux des situations de formation dans les contextes de travail avec de multiples participants.

In Education and Care professions, vocational training frequently occurs in interactions involving multiple participants. Novice workers therefore have to learn to participate in ongoing activities by engaging in work teams and by adjusting to the targeted recipients of the service. At the crossroads of research on work-based learning and interactional perspectives on work analysis, the paper proposes an empirical exploration of the forms of participation of a trainee in childhood education, during an educational activity conducted with a group of children, in the presence of two experienced educators. The notion of Participatory Configuration provides insights on how the different participants establish a definition of the situation and adapt their focus when performing collective activities. In addition, relational positioning occurs through the forms used to address the recipients and the phenomena of facework. At the epistemic level, interactants co-construct shared meaning, made mutually manifest in the interactional flow. Constant changes are part of a dynamic interaction sequence, organized sequentially and simultaneously. Knowing how to participate is therefore part of pragmatic, relational, epistemic and dynamic dimensions of interactions. This interactional perspective aims to shed light on the issues of training situations in workplace contexts with multiple participants.

\section{INDEX}

Keywords : workplace learning, interactional analysis, participatory configuration, early childhood education

Mots-clés : formation en situation de travail, analyse interactionnelle, configuration de participation, éducation de l'enfance

\section{AUTEUR}

\section{MARIANNE ZOGMAL}

Université de Genève, Équipe Interaction \& Formation, 40, Bd. Pont-d'Arve, 1211 Genève, zogmal@sunrise.ch 